\title{
Fixed point solutions for variational inequalities in image restoration over $q$-uniformly smooth Banach spaces
}

\author{
Pongsakorn Sunthrayuth and Poom Kumam*
}

\author{
${ }^{*}$ Correspondence: \\ poom.kum@kmutt.ac.th \\ Department of Mathematics, \\ Faculty of Science, King Mongkut's \\ University of Technology Thonburi \\ (KMUTT), 126 Pracha Uthit Rd., Bang \\ Mod, Thung Khru, Bangkok, 10140, \\ Thailand
}

\begin{abstract}
In this paper, we introduce new implicit and explicit iterative methods for finding a common fixed point set of an infinite family of strict pseudo-contractions by the sunny nonexpansive retractions in a real q-uniformly and uniformly convex Banach space which admits a weakly sequentially continuous generalized duality mapping. Then we prove the strong convergence under mild conditions of the purposed iterative scheme to a common fixed point of an infinite family of strict pseudo-contractions which is a solution of some variational inequalities. Furthermore, we apply our results to study some strong convergence theorems in $L_{p}$ and $\ell_{p}$ spaces with $1<p<\infty$. Our results mainly improve and extend the results announced by Ceng et al. (Comput. Math. Appl. 61:2447-2455, 2011) and many authors from Hilbert spaces to Banach spaces. Finally, we give some numerical examples for support our main theorem in the end of the paper.
\end{abstract}

MSC: 47H09; 47H10; 47H17; 47J25; 49J40

Keywords: variational inequality; Banach space; strong convergence; iterative method; common fixed point; strongly accretive operator; inverse strongly accretive operator

\section{Introduction}

Let $C_{1}, C_{2}, \ldots, C_{n}$ be nonempty, closed, and convex subsets of a real Hilbert space $H$ such that $\bigcap_{i=1}^{n} C_{i} \neq \emptyset$. The problem of image recovery in a Hilbert space setting by using convex of metric projections $P_{C_{i}}$, may be stated as follows: the original unknown image $z$ is known a priori to belong to the intersection of $\left\{C_{i}\right\}_{i=1}^{n}$; given only the metric projections $P_{C_{i}}$ of $H$ onto $C_{i}$ for $i=1,2, \ldots, n$ recover $z$ by an iterative scheme. Youla and Webb [1] first used iterative methods for applied in image restoration. The problems of image recovery have been studied in a Banach space setting by Kitahara and Takahashi [2] (see also [3, 4]) by using convex combinations of sunny nonexpansive retractions in uniformly convex Banach spaces. On the other hand, Alber [5] studied the problem of image recovery by the products of generalized projections in a uniformly convex and uniformly smooth Banach space whose duality mapping is weakly sequentially continuous (see also [6, 7]). Nakajo et al. [8] and Kimura et al. [9] considered this problem by the sunny nonexpansive retractions and proved convergence of the iterative sequence to a common point of countable nonempty, closed, and convex subsets in a uniformly convex and smooth Banach space,

(O2014 Sunthrayuth and Kumam; licensee Springer. This is an Open Access article distributed under the terms of the Creative Commons Attribution License (http://creativecommons.org/licenses/by/2.0), which permits unrestricted use, distribution, and reproduction in any medium, provided the original work is properly cited. 
and in a strictly convex, smooth and reflexive Banach space having the Kadec-Klee property, respectively. Some iterative methods have been studied in problem of image recovery by numerous authors (see $[2-5,10-12]$ ).

The problems of image recovery are connected with the convex feasibility problem, convex minimization problems, multiple-set split feasibility problems, common fixed point problems, and variational inequalities. In particular, variational inequality theory has been studied widely in several branches of pure and applied sciences. This field is dynamics and is experiencing an explosive growth in both theory and applications. Indeed, applications of the variational inequalities span as diverse disciplines as differential equations, time-optimal control, optimization, mathematical programming, mechanics, finance, and so on. Note that most of the variational problems, including minimization or maximization of functions, variational inequality problems, quasivariational inequality problems, decision and management sciences, and engineering sciences problems. Recently, some iterative methods have been developed for solving the fixed point problems and variational inequality problems in $q$-uniformly smooth Banach spaces by numerous authors (see [13-24]).

Let $A$ be a strongly positive bounded linear operator on $H$, that is, there exists a constant $\bar{\gamma}>0$ such that

$$
\langle A x, x\rangle \geq \bar{\gamma}\|x\|^{2} \quad \text { for all } x \in H \text {. }
$$

Remark 1.1 From the definition of operator $A$, we note that a strongly positive bounded linear operator $A$ is a $\|A\|$-Lipschitzian and $\eta$-strongly monotone operator.

A typical problem is to minimize a quadratic function over the set of the fixed points of a nonexpansive mapping on a real Hilbert space $H$ :

$$
\min _{x \in C} \frac{1}{2}\langle A x, x\rangle-\langle x, u\rangle
$$

where $C$ is the fixed point set of a nonexpansive mapping $T$ on $H$ and $u$ is a given point in $H$.

In 2006, Marino and $\mathrm{Xu}$ [25] introduced and considered the following a general iterative method:

$$
x_{n+1}=\alpha_{n} \gamma f\left(x_{n}\right)+\left(I-\alpha_{n} A\right) T x_{n}, \quad \forall n \geq 0,
$$

where $A$ is a strongly positive bounded linear operator on a real Hilbert space $H$. They proved that if the sequence $\left\{\alpha_{n}\right\}$ satisfies appropriate conditions, then the sequence $\left\{x_{n}\right\}$ generated by (1.3) converges strongly to the unique solution of the variational inequality

$$
\left\langle(\gamma f-A) x^{*}, x-x^{*}\right\rangle \leq 0, \quad \forall x \in \operatorname{Fix}(T),
$$

which is the optimality condition for the minimization problem

$$
\min _{x \in C} \frac{1}{2}\langle A x, x\rangle-h(x)
$$


where $C$ is the fixed point set of a nonexpansive mapping $T$ and $h$ is a potential function for $\gamma f$ (i.e., $h^{\prime}(x)=\gamma f(x)$ for all $\left.x \in H\right)$.

On the other hand, Yamada [26] introduced a hybrid steepest descent method for a nonexpansive mapping $T$ as follows:

$$
x_{n+1}=T x_{n}-\mu \lambda_{n} F\left(T x_{n}\right), \quad \forall n \geq 0,
$$

where $F$ is a $\kappa$-Lipschitzian and $\eta$-strongly monotone operator on a real Hilbert space $H$ with constants $\kappa, \eta>0$ and $0<\mu<\frac{2 \eta}{\kappa^{2}}$. He proved that if $\left\{\lambda_{n}\right\}$ satisfy the appropriate conditions, then the sequence $\left\{x_{n}\right\}$ generated by (1.6) converges strongly to the unique solution of the variational inequality

$$
\left\langle F x^{*}, x-x^{*}\right\rangle \geq 0, \quad \forall x \in \operatorname{Fix}(T) .
$$

Tian [27] combined the iterative method (1.3) with the Yamada method (1.6) and considered a general iterative method for a nonexpansive mapping $T$ on a real Hilbert space $H$ as follows:

$$
x_{n+1}=\alpha_{n} \gamma f\left(x_{n}\right)+\left(I-\alpha_{n} \mu F\right) T x_{n}, \quad \forall n \geq 0 .
$$

Then he proved that the sequence $\left\{x_{n}\right\}$ generated by (1.8) converges strongly to the unique solution of variational inequality

$$
\left\langle(\gamma f-\mu F) x^{*}, x-x^{*}\right\rangle \leq 0, \quad \forall x \in \operatorname{Fix}(T)
$$

In 2011, Ceng et al. [28] combined the iterative method (1.3) with Tian's method (1.8) and consider the following a general composite iterative method:

$$
x_{n+1}=\left(I-\alpha_{n} A\right) T x_{n}+\alpha_{n}\left[T x_{n}-\beta_{n}\left(\mu F T x_{n}-\gamma f\left(x_{n}\right)\right)\right], \quad \forall n \geq 0,
$$

where $A$ is a strongly positive bounded linear operator on $H$ with coefficient $\bar{\gamma} \in(1,2)$, and $\left\{\alpha_{n}\right\} \subset(0,1)$ and $\left\{\beta_{n}\right\} \subset(0,1]$ satisfy appropriate conditions. Then they proved that the sequence $\left\{x_{n}\right\}$ generated by (1.10) converges strongly to the unique solution $x^{*} \in C$ of the variational inequality

$$
\left\langle(I-A) x^{*}, x-x^{*}\right\rangle \leq 0, \quad \forall x \in C,
$$

where $C=\operatorname{Fix}(T)$.

In this paper, motivated by the above facts, we introduce new implicit and explicit iterative methods for finding a common fixed point set of an infinite family of strict pseudocontractions by the sunny nonexpansive retractions in a real $q$-uniformly and uniformly convex Banach space $X$ which admits a weakly sequentially continuous generalized duality mapping. Consequently, we prove the strong convergence under mild conditions of the purposed iterative scheme to a common fixed point of an infinite family of strict pseudocontractions of nonempty, closed, and convex subsets of $X$ which is a solution of some variational inequalities. Furthermore, we apply our results to the study of some strong 
convergence theorems in $L_{p}$ and $\ell_{p}$ spaces with $1<p<\infty$. Our results extend the main result of Ceng et al. [28] in several aspects and the work of many authors from Hilbert spaces to Banach spaces. Finally, we give some numerical examples to support our main theorem in the end of the paper.

\section{Preliminaries}

Throughout this paper, we denote by $X$ and $X^{*}$ a real Banach space and the dual space of $X$, respectively. Let $q>1$ be a real number. The generalized duality mapping $J_{q}: X \rightarrow 2^{X^{*}}$ is defined by

$$
J_{q}(x)=\left\{f \in X^{*}:\langle x, f\rangle=\|x\|^{q},\|f\|=\|x\|^{q-1}\right\},
$$

where $\langle\cdot, \cdot\rangle$ denotes the duality pairing between $X$ and $X^{*}$. In particular, $J_{q}=J_{2}$ is called the normalized duality mapping and $J_{q}(x)=\|x\|^{q-2} J_{2}(x)$ for $x \neq 0$. If $X:=H$ is a real Hilbert space, then $J=I$, where $I$ is the identity mapping. It is well known that if $X$ is smooth, then $J_{q}$ is single-valued, which is denoted by $j_{q}$ (see [29]).

A Banach space $X$ is said to be strictly convex if $\frac{\|x+y\|}{2}<1$ for all $x, y \in X$ with $\|x\|=\|y\|=1$ and $x \neq y$. A Banach space $X$ is said to be uniformly convex if, for each $\epsilon>0$, there exists $\delta>0$ such that for $x, y \in X$ with $\|x\|,\|y\| \leq 1$ and $\|x-y\| \geq \epsilon, \frac{\|x+y\|}{2} \leq 1-\delta$ holds. Let $S(X)=\{x \in X:\|x\|=1\}$. The norm of $X$ is said to be Gâteaux differentiable (or $X$ is said to be smooth) if the limit

$$
\lim _{t \rightarrow 0} \frac{\|x+t y\|-\|x\|}{t}
$$

exists for each $x, y \in S(X)$. The norm of $X$ is said to be uniformly Gâteaux differentiable, if, for each $y \in S(X)$, the limit is attained uniformly for $x \in S(X)$.

Let $\rho_{X}:[0, \infty) \rightarrow[0, \infty)$ be the modulus of smoothness of $X$ defined by

$$
\rho_{X}(\tau)=\sup \left\{\frac{1}{2}(\|x+y\|+\|x-y\|)-1: x \in S(X),\|y\| \leq \tau\right\} .
$$

A Banach space $X$ is said to be uniformly smooth if $\frac{\rho_{X}(t)}{t} \rightarrow 0$ as $t \rightarrow 0$. Suppose that $q>1$, then $X$ is said to be q-uniformly smooth if there exists $c>0$ such that $\rho_{X}(t) \leq c t^{q}$ for all $t>0$. It is shown in [30] (see also [31]) that there is no Banach space which is $q$-uniformly smooth with $q>2$. If $X$ is $q$-uniformly smooth, then $X$ is uniformly smooth. It is well known that each uniformly convex Banach space $X$ is reflexive and strictly convex and every uniformly smooth Banach space $X$ is a reflexive Banach space with uniformly Gâteaux differentiable norm (see [29]). Typical examples of both uniformly convex and uniformly smooth Banach spaces are $L_{p}$, where $p>1$. More precisely, $L_{p}$ is $\min \{p, 2\}$-uniformly smooth for every $p>1$.

Let $C$ be a nonempty, closed, and convex subset of $X$ and $T$ be a self-mapping on $C$. We denote the fixed points set of the mapping $T$ by $\operatorname{Fix}(T)=\{x \in C: T x=x\}$.

Definition 2.1 A mapping $T: C \rightarrow C$ is said to be:

(i) $\lambda$-strictly pseudo-contractive [32] if, for all $x, y \in C$, there exist $\lambda>0$ and $j_{q}(x-y) \in J_{q}(x-y)$ such that

$$
\left\langle T x-T y, j_{q}(x-y)\right\rangle \leq\|x-y\|^{q}-\lambda\|(I-T) x-(I-T) y\|^{q},
$$


or equivalently

$$
\left\langle(I-T) x-(I-T) y, j_{q}(x-y)\right\rangle \geq \lambda\|(I-T) x-(I-T) y\|^{q} .
$$

(ii) L-Lipschitzian if, for all $x, y \in C$, there exists a constant $L>0$ such that

$$
\|T x-T y\| \leq L\|x-y\| .
$$

If $0<L<1$, then $T$ is a contraction and if $L=1$, then $T$ is a nonexpansive mapping. By the definition, we know that every $\lambda$-strictly pseudo-contractive mapping is $\left(\frac{1+\lambda}{\lambda}\right)$-Lipschitzian (see [33])

Remark 2.2 Let $C$ be a nonempty subset of a real Hilbert space $H$ and $T: C \rightarrow C$ be a mapping. Then $T$ is said to be $k$-strictly pseudo-contractive [32] if, for all $x, y \in C$, there exists $k \in[0,1)$ such that

$$
\|T x-T y\|^{2} \leq\|x-y\|^{2}+k\|(I-T) x-(I-T) y\|^{2} .
$$

It is well known that (2.3) is equivalent to the following inequality:

$$
\langle T x-T y, x-y\rangle \leq\|x-y\|^{2}-\frac{1-k}{2}\|(I-T) x-(I-T) y\|^{2} .
$$

A mapping $F: C \rightarrow X$ is said to be accretive if, for all $x, y \in C$, there exists $j_{q}(x-y) \in$ $J_{q}(x-y)$ such that

$$
\left\langle F x-F y, j_{q}(x-y)\right\rangle \geq 0
$$

For some $\eta>0, F: C \rightarrow X$ is said to be strongly accretive if, for all $x, y \in C$, there exists $j_{q}(x-y) \in J_{q}(x-y)$ such that

$$
\left\langle F x-F y, j_{q}(x-y)\right\rangle \geq \eta\|x-y\|^{q} .
$$

Remark 2.3 If $X:=H$ is a real Hilbert space, accretive and strongly accretive mappings coincide with monotone and strongly monotone mappings, respectively.

Let $D$ be a nonempty subset of $C$. A mapping $Q: C \rightarrow D$ is said to be sunny [34] if

$$
Q(Q x+t(x-Q x))=Q x,
$$

whenever $Q x+t(x-Q x) \in C$ for $x \in C$ and $t \geq 0$. A mapping $Q: C \rightarrow D$ is said to be retraction if $Q x=x$ for all $x \in D$. Furthermore, $Q$ is a sunny nonexpansive retraction from $C$ onto $D$ if $Q$ is a retraction from $C$ onto $D$ which is also sunny and nonexpansive. A subset $D$ of $C$ is called a sunny nonexpansive retraction of $C$ if there exists a sunny nonexpansive retraction from $C$ onto $D$. It is well known that if $X:=H$ is a real Hilbert space, then a sunny nonexpansive retraction $Q$ is coincident with the metric projection from $X$ onto $C$.

Lemma 2.4 ([14]) Let $C$ be a closed and convex subset of a smooth Banach space $X$. Let $D$ be a nonempty subset of $C$. Let $Q: C \rightarrow D$ be a retraction and let $j, j_{q}$ be the normalized 
duality mapping and generalized duality mapping on $X$, respectively. Then the following are equivalent:

(a) $Q$ is sunny and nonexpansive.

(b) $\|Q x-Q y\|^{2} \leq\langle x-y, j(Q x-Q y)\rangle$ for all $x, y \in C$.

(c) $\langle x-Q x, j(y-Q x)\rangle \leq 0$ for all $x \in C$ and $y \in D$.

(d) $\left\langle x-Q x, j_{q}(y-Q x)\right\rangle \leq 0$ for all $x \in C$ and $y \in D$.

Lemma 2.5 ([35]) Suppose that $q>1$. Then the following inequality holds:

$$
a b \leq \frac{1}{q} a^{q}+\left(\frac{q-1}{q}\right) b^{\frac{q}{q-1}}
$$

for arbitrary positive real numbers $a, b$.

In a real $q$-uniformly smooth Banach space, Xu [36] proved the following important inequality:

Lemma 2.6 ([36]) Let $X$ be a real q-uniformly smooth Banach space. Then the following inequality holds:

$$
\|x+y\|^{q} \leq\|x\|^{q}+q\left\langle y, J_{q}(x)\right\rangle+C_{q}\|y\|^{q}
$$

for all $x, y \in X$ and for some $C_{q}>0$.

Remark 2.7 The constant $C_{q}$ satisfying (2.4) is called the best q-uniform smoothness constant.

Lemma 2.8 ([21]) Let $C$ be a nonempty and convex subset of a real q-uniformly smooth Banach space $X$ and $T: C \rightarrow C$ be a $\lambda$-strict pseudo-contraction. For $\gamma \in(0,1)$, define $S x=(1-\gamma) x+\gamma T x$. Then, as $\gamma \in(0, v), v=\min \left\{1,\left(\frac{q \lambda}{C_{q}}\right)^{\frac{1}{q-1}}\right\}, S: C \rightarrow C$ is nonexpansive and $\operatorname{Fix}(S)=\operatorname{Fix}(T)$, where $C_{q}$ is the best q-uniform smoothness constant.

Definition 2.9 ([37]) Let $C$ be a nonempty, closed, and convex subset of a real $q$-uniformly smooth Banach space $X$. Let $T_{n, k}=\theta_{n, k} S_{k}+\left(1-\theta_{n, k}\right) I$, where $S_{k}: C \rightarrow C$ is $\lambda_{k}$-strict pseudocontraction and $\left\{t_{n}\right\}$ be a nonnegative real sequence with $0 \leq t_{n} \leq 1, \forall n \in \mathbb{N}$. For $n \geq 1$, define a mapping $W_{n}: C \rightarrow C$ as follows:

$$
\begin{aligned}
& U_{n, n+1}=I, \\
& U_{n, n}=t_{n} T_{n, n} U_{n, n+1}+\left(1-t_{n}\right) I, \\
& \vdots \\
& U_{n, k}=t_{k} T_{n, k} U_{n, k+1}+\left(1-t_{k}\right) I, \\
& U_{n, k-1}=t_{k-1} T_{n, k-1} U_{n, k}+\left(1-t_{k-1}\right) I, \\
& \vdots \\
& U_{n, 2}=t_{2} T_{n, 2} U_{n, 3}+\left(1-t_{2}\right) I, \\
& W_{n}=U_{n, 1}=t_{1} T_{n, 1} U_{n, 2}+\left(1-t_{1}\right) I .
\end{aligned}
$$


Such a mapping $W_{n}$ is called the $W$-mapping generated by $T_{n, n}, T_{n, n-1}, \ldots, T_{n, 1}$ and $t_{n}, t_{n-1}$, $\ldots, t_{1}$.

Throughout this paper, we will assume that $\left\{\theta_{n, k}\right\}$ satisfies the following conditions:

(H1) $\theta_{n, k} \in(0, v], v=\min \left\{1,\left(\frac{q \bar{\lambda}}{C_{q}}\right)^{\frac{1}{q-1}}\right\}$ with $\bar{\lambda}=\inf \lambda_{k}>0, \forall n, k \in \mathbb{N}$;

(H2) $\left|\theta_{n+1, k}-\theta_{n, k}\right| \leq a_{n}, \forall n \in \mathbb{N}$ and $1 \leq k \leq n$ with $\sum_{n=1}^{\infty} a_{n}<\infty$;

The hypothesis $(\mathrm{H} 2)$ secures the existence of $\lim _{n \rightarrow \infty} \theta_{n, k}, \forall k \in \mathbb{N}$. Set $\theta_{1, k}:=\lim _{n \rightarrow \infty} \theta_{n, k}$, $\forall n \in \mathbb{N}$. Furthermore, we assume

(H3) $\theta_{1, k}>0, \forall k \in \mathbb{N}$.

It is obvious that $\theta_{1, k}$ satisfies (H1). Using condition (H3), from $T_{n, k}=\theta_{n, k} S_{k}+\left(1-\theta_{n, k}\right) I$, we define mappings $T_{1, k} x:=\lim _{n \rightarrow \infty} T_{n, k} x=\theta_{1, k} S_{k} x+\left(1-\theta_{1, k}\right) x, \forall x \in C$.

Lemma 2.10 ([37]) Let $C$ be a nonempty, closed, and convex subset of a real q-uniformly smooth and strictly convex Banach space X. Let $T_{n, i}=\theta_{n, i} S_{i}+\left(1-\theta_{n, i}\right) I$, where $S_{i}: C \rightarrow C$ $(i=1,2, \ldots)$ is $\lambda_{i}$-strict pseudo-contraction with $\bigcap_{n=1}^{\infty} \operatorname{Fix}\left(S_{n}\right) \neq \emptyset$ and $\inf \lambda_{i}>0$. Let $t_{1}, t_{2}, \ldots$ be nonnegative real numbers such that $0<t_{n} \leq b<1, \forall n \geq 1$. Assume the sequence $\left\{\theta_{n, k}\right\}$ satisfies (H1)-(H3). Then

(1) $W_{n}$ is nonexpansive and $\operatorname{Fix}\left(W_{n}\right)=\bigcap_{n=1}^{\infty} \operatorname{Fix}\left(S_{n}\right)$ for each $n \geq 1$;

(2) for each $x \in C$ and for each positive integer $k$, the limit $\lim _{n \rightarrow \infty} U_{n, k}$ exists;

(3) the mapping $W: C \rightarrow C$ defined by

$$
W x:=\lim _{n \rightarrow \infty} W_{n} x=\lim _{n \rightarrow \infty} U_{n, 1} x, \quad \forall x \in C,
$$

is a nonexpansive mapping satisfying $\operatorname{Fix}(W)=\bigcap_{n=1}^{\infty} \operatorname{Fix}\left(S_{n}\right)$ and it is called the $W$-mapping generated by $S_{1}, S_{2}, \ldots$ and $t_{1}, t_{2}, \ldots$ and $\theta_{n, k}, \forall n \in \mathbb{N}$ and $1 \leq k \leq n$.

Lemma 2.11 ([37]) Let $C$ be a nonempty, closed, and convex subset of a real q-uniformly smooth and strictly convex Banach space $X$. Let $T_{n, i}=\theta_{n, i} S_{i}+\left(1-\theta_{n, i}\right) I$, where $S_{i}: C \rightarrow C$ $(i=1,2, \ldots)$ is $\lambda_{i}$-strict pseudo-contraction with $\bigcap_{n=1}^{\infty} \operatorname{Fix}\left(S_{n}\right) \neq \emptyset$ and inf $\lambda_{i}>0$. Let $t_{1}, t_{2}, \ldots$ be nonnegative real numbers such that $0<t_{n} \leq b<1, \forall n \geq 1$. Assume the sequence $\left\{\theta_{n, k}\right\}$ satisfies (H1)-(H3). If $\left\{\omega_{n}\right\}$ is a bounded sequence in $C$, then

$$
\lim _{n \rightarrow \infty}\left\|W \omega_{n}-W_{n} \omega_{n}\right\|=0
$$

In the following, the notation $\rightarrow$ and $\rightarrow$ denote the weak and strong convergence, respectively. The duality mapping $J_{q}$ from a smooth Banach space $X$ into $X^{*}$ is said to be weakly sequentially continuous generalized duality mapping if, for all $\left\{x_{n}\right\} \subset X, x_{n} \rightarrow x$ implies $J_{q}\left(x_{n}\right) \rightarrow^{*} J_{q}(x)$.

A Banach space $X$ is said to be satisfy Opial's condition [38], that is, for any sequence $\left\{x_{n}\right\}$ in $X, x_{n} \rightarrow x$ implies that

$$
\liminf _{n \rightarrow \infty}\left\|x_{n}-x\right\|<\liminf _{n \rightarrow \infty}\left\|x_{n}-y\right\|, \quad \forall y \in X \text { with } x \neq y .
$$

By Theorem 3.2.8 in [39], it is well known that if $X$ admits a weakly sequentially continuous generalized duality mapping, then $X$ satisfies Opial's condition.

Lemma 2.12 ([13]) Let $C$ be a nonempty, closed, and convex subset of a real q-uniformly smooth Banach space $X$ which admits weakly sequentially continuous generalized duality 
mapping $j_{q}$ from $X$ into $X^{*}$. Let $T: C \rightarrow C$ be a nonexpansive mapping. Then, for all $\left\{x_{n}\right\} \subset$ $C$, if $x_{n} \rightarrow x$ and $x_{n}-T x_{n} \rightarrow 0$, then $x=T x$.

Lemma 2.13([40]) Let $\left\{a_{n}\right\},\left\{\mu_{n}\right\}$, and $\left\{\delta_{n}\right\}$ be real sequences of nonnegative numbers such that

$$
a_{n+1} \leq\left(1-\sigma_{n}\right) a_{n}+\mu_{n}+\delta_{n}, \quad \forall n \geq 1,
$$

where $\sigma_{n} \in(0,1), \sum_{n=1}^{\infty} \sigma_{n}=\infty, \mu_{n}=\circ\left(\sigma_{n}\right)$ and $\sum_{n=1}^{\infty} \delta_{n}<\infty$. Then $\lim _{n \rightarrow \infty} a_{n}=0$.

\section{Main results}

In order to prove our main result, the following lemma is needed.

Lemma 3.1 Let $C$ be a nonempty, closed, and convex subset of a real q-uniformly smooth Banach space $X$ with the best q-uniform smoothness constant $C_{q}>0$. Let $F: C \rightarrow X$ be a $\kappa$-Lipschitzian and $\eta$-strongly accretive operator with constants $\kappa, \eta>0$. Let $0<\mu<$ $\left(\frac{q \eta}{C_{q} \kappa^{q}}\right)^{\frac{1}{q-1}}$ and $\tau=\mu\left(\eta-\frac{C_{q} \mu^{q-1} \kappa^{q}}{q}\right)$. Then for $t \in\left(0, \min \left\{1, \frac{1}{q \tau}\right\}\right)$, the mapping $S: C \rightarrow X d e-$ fined by $S:=I-t \mu F$ is a contraction with constant $1-t \tau$.

Proof Since $0<\mu<\left(\frac{q \eta}{C_{q} \kappa^{q}}\right)^{\frac{1}{q-1}}$ and $t \in\left(0, \min \left\{1, \frac{1}{q \tau}\right\}\right)$. This implies that $1-t \tau \in(0,1)$. From Lemma 2.6, for all $x, y \in C$, we have

$$
\begin{aligned}
\|S x-S y\|^{q} & =\|(I-t \mu F) x-(I-t \mu F) y\|^{q} \\
& =\|(x-y)-t \mu(F x-F y)\|^{q} \\
& \leq\|x-y\|^{q}-q t \mu\left\langle F x-F y, j_{q}(x-y)\right\rangle+C_{q} t^{q} \mu^{q}\|F x-F y\|^{q} \\
& \leq\|x-y\|^{q}-q t \mu \eta\|x-y\|^{q}+C_{q} t^{q} \mu^{q} \kappa^{q}\|x-y\|^{q} \\
& \leq\left[1-t \mu\left(q \eta-C_{q} \mu^{q-1} \kappa^{q}\right)\right]\|x-y\|^{q} \\
& =\left[1-t \mu q\left(\eta-\frac{C_{q} \mu^{q-1} \kappa^{q}}{q}\right)\right]\|x-y\|^{q} \\
& \leq\left[1-t \mu\left(\eta-\frac{C_{q} \mu^{q-1} \kappa^{q}}{q}\right)\right]^{q}\|x-y\|^{q} \\
& =(1-t \tau)^{q}\|x-y\|^{q} .
\end{aligned}
$$

It follows that

$$
\|S x-S y\| \leq(1-t \tau)\|x-y\| .
$$

Hence, we have $S:=I-t \mu F$ is a contraction with constant $1-t \tau$.

Lemma 3.2 Let $C$ be a nonempty, closed, and convex subset of a real q-uniformly smooth Banach space $X$ and $G: C \rightarrow X$ be a mapping.

(i) If $G$ is a $\delta$-strongly accretive and $\lambda$-strictly pseudo-contractive mapping wit $\delta+\lambda>1$, then $I-G$ is a contraction with constant $L_{\delta, \lambda}:=\left(\frac{1-\delta}{\lambda}\right)^{\frac{1}{q}}$.

(ii) If $G$ is a $\delta$-strongly accretive and $\lambda$-strictly pseudo-contractive mapping with $\delta+\lambda>1$. For a fixed number $t \in(0,1)$, then $I-t G$ is a contraction with constant $1-\left(1-L_{\delta, \lambda}\right) t$. 
Proof (i) For all $x, y \in C$, from (2.2), we have

$$
\begin{aligned}
\lambda\|(I-G) x-(I-G) y\|^{q} & \leq\|x-y\|^{q}-\left\langle G x-G y, j_{q}(x-y)\right\rangle \\
& \leq(1-\delta)\|x-y\|^{q} .
\end{aligned}
$$

Observe that

$$
\delta+\lambda>1 \Longleftrightarrow\left(\frac{1-\delta}{\lambda}\right)^{\frac{1}{q}} \in(0,1) .
$$

It follows that

$$
\|(I-G) x-(I-G) y\| \leq\left(\frac{1-\delta}{\lambda}\right)^{\frac{1}{q}}\|x-y\|:=L_{\delta, \lambda}\|x-y\| .
$$

Hence, $I-G$ is a contraction with constant $L_{\delta, \lambda}$.

(ii) Since $I-G$ is a contraction with constant $L_{\delta, \lambda}$. For all $t \in(0,1)$, we have

$$
\begin{aligned}
\|(I-t G) x-(I-t G) y\| & =\|(x-y)-t(G x-G y)\| \\
& =\|(1-t)(x-y)+t[(I-G) x-(I-G) y]\| \\
& \leq(1-t)\|x-y\|+t\|(I-G) x-(I-G) y\| \\
& \leq\left(1-\left(1-L_{\delta, \lambda}\right) t\right)\|x-y\| .
\end{aligned}
$$

Hence, $I-t G$ is a contraction with constant $1-\left(1-L_{\delta, \lambda}\right) t$. This completes the proof.

\subsection{Implicit iteration scheme}

Let $C$ be a nonempty, closed, and convex subset of a real reflexive and $q$-uniformly smooth Banach space $X$ which admits a weakly sequentially continuous generalized duality mapping $j_{q}$. Let $Q_{C}$ be a sunny nonexpansive retraction from $X$ onto $C$. Let $F: C \rightarrow X$ be a $\kappa$-Lipschitzian and $\eta$-strongly accretive operator with constants $\kappa, \eta>0, G: C \rightarrow X$ be a $\delta$-strongly accretive and $\lambda$-strictly pseudo-contractive mapping with $\delta+\lambda>1, V: C \rightarrow X$ be an $L$-Lipschitzian mapping with constant $L \geq 0$ and $T: C \rightarrow C$ be a nonexpansive mapping such that $\operatorname{Fix}(T) \neq \emptyset$. Let $0<\mu<\left(\frac{q \eta}{C_{q^{k}}}\right)^{\frac{1}{q-1}}$ and $0 \leq \gamma L<\tau$, where $\tau=\mu\left(\eta-\frac{C_{q} \mu^{q-1} \kappa^{q}}{q}\right)$. For each $\sigma \in\left(\frac{L_{\delta, \lambda}}{\tau-\gamma L}, \min \left\{1, \frac{1}{q \tau}, \frac{1+L_{\delta, \lambda}}{\tau-\gamma L}\right\}\right)$ and $t \in(0,1)$, we define a mapping $S_{t}: C \rightarrow C$ defined by

$$
S x:=Q_{C}[(I-t G) T x+t(T x-\sigma(\mu F T x-\gamma V x))], \quad \forall x \in C .
$$

It is easy to see immediately that $S_{t}$ is a contraction. Indeed, for all $x, y \in C$, from Lemmas 3.1 and 3.2(ii), we have

$$
\begin{aligned}
\left\|S_{t} x-S_{t} y\right\|= & \| Q_{C}[(I-t G) T x+t(T x-\sigma(\mu F T x-\gamma V x))] \\
& -Q_{C}[(I-t G) T y+t(T y-\sigma(\mu F T y-\gamma V y))] \| \\
\leq & \|(I-t G)(T x-T y)+t[(I-\sigma \mu F)(T x-T y)+\sigma \gamma(V x-V y)]\|
\end{aligned}
$$




$$
\begin{aligned}
& \leq\left(1-t\left(1-L_{\delta, \lambda}\right)\right)\|x-y\|+t[\sigma \gamma\|V x-V y\|+\|(I-\sigma \mu F)(T x-T y)\|] \\
& \leq\left(1-t\left(1-L_{\delta, \lambda}\right)\right)\|x-y\|+t(1-\sigma(\tau-\gamma L))\|x-y\| \\
& =\left[1-t\left(\sigma(\tau-\gamma L)-L_{\delta, \lambda}\right)\right]\|x-y\| \\
& =(1-t \theta)\|x-y\|,
\end{aligned}
$$

where $\theta:=\sigma(\tau-\gamma L)-L_{\delta, \lambda}$. Since $\tau-\gamma L>0$ and $L_{\delta, \lambda} \in(0,1)$, observe that

$$
\frac{L_{\delta, \lambda}}{\tau-\gamma L}<\sigma<\min \left\{1, \frac{1}{q \tau}, \frac{1+L_{\delta, \lambda}}{\tau-\gamma L}\right\} \leq \frac{1+L_{\delta, \lambda}}{\tau-\gamma L} .
$$

It follows that

$$
\sigma<\frac{1+L_{\delta, \lambda}}{\tau-\gamma L} \quad \Longleftrightarrow \quad \theta=\sigma(\tau-\gamma L)-L_{\delta, \lambda}<1
$$

and

$$
\frac{L_{\delta, \lambda}}{\tau-\gamma L}<\sigma \quad \Longleftrightarrow \quad \theta=\sigma(\tau-\gamma L)-L_{\delta, \lambda}>0 .
$$

This implies that $\theta=\sigma(\tau-\gamma L)-L_{\delta, \lambda} \in(0,1)$, which together with $t \in(0,1)$ gives

$$
1-t\left(\sigma(\tau-\gamma L)-L_{\delta, \lambda}\right) \in(0,1)
$$

Hence $S_{t}$ is a contraction. By the Banach contraction principle, $S_{t}$ has a unique fixed point, denote by $x_{t}$, which uniquely solves the fixed point equation

$$
x_{t}=Q_{C}\left[(I-t G) T x_{t}+t\left(T x_{t}-\sigma\left(\mu F T x_{t}-\gamma V x_{t}\right)\right)\right] .
$$

The following proposition summarizes the properties of the net $\left\{x_{t}\right\}$.

Proposition 3.3 Let $\left\{x_{t}\right\}$ be defined by (3.2). Then the following hold:

(i) $\left\{x_{t}\right\}$ is bounded for each $t \in(0,1)$;

(ii) $\lim _{t \rightarrow 0}\left\|x_{t}-T x_{t}\right\|=0$;

(iii) $\left\{x_{t}\right\}$ defines a continuous curve from $(0,1)$ into $C$.

Proof (i) Take $p \in \operatorname{Fix}(T)$, and denote a mapping $S_{t}: C \rightarrow C$ by

$$
S_{t} x:=Q_{C}[(I-t G) T x+t(T x-\sigma(\mu F T x-\gamma V x))], \quad \forall x \in C .
$$

From (3.1), we have

$$
\begin{aligned}
\left\|x_{t}-p\right\| & \leq\left\|S_{t} x_{t}-S_{t} p\right\|+\left\|S_{t} p-p\right\| \\
& \leq(1-t \theta)\left\|x_{t}-p\right\|+\left\|Q_{C}[(I-t G) T p+t(T p-\sigma(\mu F T p-\gamma V p))]-Q_{C} p\right\| \\
& \leq(1-t \theta)\left\|x_{t}-p\right\|+t\|-G p+p-\sigma(\mu F p-\gamma V p)\| \\
& \leq(1-t \theta)\left\|x_{t}-p\right\|+t[\|I-G\|\|p\|+\sigma \mu\|F p\|+\sigma \gamma\|V p\|],
\end{aligned}
$$


where $\theta:=\sigma(\tau-\gamma L)-L_{\delta, \lambda}$. It follows that

$$
\left\|x_{t}-p\right\| \leq \frac{\|I-G\|\|p\|+\sigma \mu\|F p\|+\sigma \gamma\|V p\|}{\theta} .
$$

Hence $\left\{x_{t}\right\}$ is bounded, so are $\left\{V x_{t}\right\},\left\{F T x_{t}\right\}$, and $\left\{G T x_{t}\right\}$.

(ii) By definition of $\left\{x_{t}\right\}$, we have

$$
\begin{aligned}
\left\|x_{t}-T x_{t}\right\| & =\left\|Q_{C}\left[(I-t G) T x_{t}+t\left(T x_{t}-\sigma\left(\mu F T x_{t}-\gamma V x_{t}\right)\right)\right]-Q_{C} T x_{t}\right\| \\
& \leq t\left\|(I-G) T x_{t}-\sigma\left(\mu F T x_{t}-\gamma V x_{t}\right)\right\| \rightarrow 0 \quad \text { as } t \rightarrow 0 .
\end{aligned}
$$

(iii) Take $t, t_{0} \in(0,1)$ and calculate

$$
\begin{aligned}
\left\|x_{t}-x_{t_{0}}\right\|= & \| Q_{C}\left[(I-t G) T x_{t}+t\left(T x_{t}-\sigma\left(\mu F T x_{t}-\gamma V x_{t}\right)\right)\right] \\
& -Q_{C}\left[\left(I-t_{0} G\right) T x_{t_{0}}+t\left(T x_{t_{0}}-\sigma\left(\mu F T x_{t_{0}}-\gamma V x_{t_{0}}\right)\right)\right] \| \\
\leq & \|\left(t_{0}-t\right) G T x_{t}+\left(I-t_{0} G\right)\left(T x_{t}-T x_{t_{0}}\right)+\left(t-t_{0}\right)\left[T x_{t}-\sigma\left(\mu F T x_{t}-\gamma V x_{t}\right)\right] \\
& +t_{0}\left[T x_{t}-\sigma\left(\mu F T x_{t_{0}}-\gamma V x_{t_{0}}\right)-\left[T x_{t_{0}}-\sigma\left(\mu F T x_{t_{0}}-\gamma V x_{t_{0}}\right)\right]\right] \| \\
= & \|\left(t_{0}-t\right) G T x_{t}+\left(I-t_{0} G\right)\left(T x_{t}-T x_{t_{0}}\right)+\left(t-t_{0}\right)\left[T x_{t}-\sigma\left(\mu F T x_{t}-\gamma V x_{t}\right)\right] \\
& +t_{0}\left[\sigma \gamma\left(V x_{t}-V x_{t_{0}}\right)+(I-\sigma \mu F)\left(T x_{t}-T x_{t_{0}}\right)\right] \| \\
\leq & \left|t-t_{0}\right|\left\|G T x_{t}\right\|+\left(1-\left(1-L_{\delta, \lambda}\right)\right)\left\|x_{t}-x_{t_{0}}\right\| \\
& +\left|t-t_{0}\right|\left\|T x_{t}-\sigma\left(\mu F T x_{t}-\gamma V x_{t}\right)\right\| \\
& +t_{0}(1-\sigma(\tau-\gamma L))\left\|x_{t}-x_{t_{0}}\right\| .
\end{aligned}
$$

It follows that

$$
\left\|x_{t}-x_{t_{0}}\right\| \leq \frac{\left\|G T x_{t}\right\|+\left\|T x_{t}-\sigma\left(\mu F T x_{t}-\gamma V x_{t}\right)\right\|}{t_{0}\left(\sigma(\tau-\gamma L)-L_{\delta, \lambda}\right)}\left|t-t_{0}\right| .
$$

Since $\left\{V x_{t}\right\},\left\{F T x_{t}\right\}$, and $\left\{G T x_{t}\right\}$ are bounded. Hence $\left\{x_{t}\right\}$ defines a continuous curve from $(0,1)$ into $C$.

Theorem 3.4 Assume that $\left\{x_{t}\right\}$ is defined by (3.2), then $\left\{x_{t}\right\}$ converges strongly to $x^{*} \in$ $\operatorname{Fix}(T)$ as $t \rightarrow 0$, where $x^{*}$ is the unique solution of the variational inequality

$$
\left\langle(G-I+\sigma(\mu F-\gamma V)) x^{*}, j_{q}\left(x^{*}-v\right)\right\rangle \leq 0, \quad \forall v \in \operatorname{Fix}(T) .
$$

Proof We observe that

$$
\begin{aligned}
\frac{C_{q} \mu^{q-1} \kappa^{q}}{q}>0 & \Longleftrightarrow \eta-\frac{C_{q} \mu^{q-1} \kappa^{q}}{q}<\eta \\
& \Longleftrightarrow \mu\left(\eta-\frac{C_{q} \mu^{q-1} \kappa^{q}}{q}\right)<\mu \eta \\
& \Longleftrightarrow \tau<\mu \eta .
\end{aligned}
$$


It follows that

$$
0 \leq \gamma L<\tau<\mu \eta
$$

First, we show the uniqueness of solution of the variational inequality. Suppose that $\tilde{x}, x^{*} \in$ $\operatorname{Fix}(T)$ are solutions of (3.3), then

$$
\left\langle(G-I+\sigma(\mu F-\gamma V)) x^{*}, j_{q}\left(x^{*}-\tilde{x}\right)\right\rangle \leq 0
$$

and

$$
\left\langle(G-I+\sigma(\mu F-\gamma V)) \tilde{x}, j_{q}\left(\tilde{x}-x^{*}\right)\right\rangle \leq 0 .
$$

Adding up (3.6) and (3.7), and from Lemma 3.2(i), we obtain

$$
\begin{aligned}
0 \geq & \left\langle(G-I+\sigma(\mu F-\gamma V)) x^{*}-(G-I+\sigma(\mu F-\gamma V)) \tilde{x}, j_{q}\left(x^{*}-\tilde{x}\right)\right\rangle \\
= & \left\langle(G-I) x^{*}-(G-I) \tilde{x}, j_{q}\left(x^{*}-\tilde{x}\right)\right\rangle+\sigma\left\langle(\mu F-\gamma V) x^{*}-(\mu F-\gamma V) \tilde{x}, j_{q}\left(x^{*}-\tilde{x}\right)\right\rangle \\
= & -\left\langle(I-G) x^{*}-(I-G) \tilde{x}, j_{q}\left(x^{*}-\tilde{x}\right)\right\rangle+\sigma \mu\left\langle F x^{*}-F \tilde{x}, j_{q}\left(x^{*}-\tilde{x}\right)\right\rangle \\
& -\sigma \gamma\left\langle V x^{*}-V \tilde{x}, j_{q}\left(x^{*}-\tilde{x}\right)\right\rangle \\
\geq & -L_{\delta, \lambda}\left\|x^{*}-\tilde{x}\right\|^{q}+\sigma \mu \eta\left\|x^{*}-\tilde{x}\right\|^{q}-\sigma \gamma\left\|V x^{*}-V \tilde{x}\right\|\left\|x^{*}-\tilde{x}\right\|^{q-1} \\
\geq & \left(\sigma(\mu \eta-\gamma L)-L_{\delta, \lambda}\right)\left\|x^{*}-\tilde{x}\right\|^{q} .
\end{aligned}
$$

On the other hand, we observe from (3.5) that

$$
\begin{aligned}
\frac{L_{\delta, \lambda}}{\tau-\gamma L}<\sigma & \Longleftrightarrow L_{\delta, \lambda}<\sigma(\tau-\gamma L) \\
& \Longleftrightarrow L_{\delta, \lambda}<\sigma(\mu \eta-\gamma L) \\
& \Longleftrightarrow 0<\sigma(\mu \eta-\gamma L)-L_{\delta, \lambda} .
\end{aligned}
$$

Note that (3.8) implies that $x^{*}=\tilde{x}$ and the uniqueness is proved. Below, we use $\tilde{x}$ to denote the unique solution of the variational inequality (3.3).

Next, we show that $x_{t} \rightarrow x^{*}$ as $t \rightarrow 0$. Set $x_{t}=Q_{C} y_{t}$, where $y_{t}=(I-t G) T x_{t}+t\left(T x_{t}-\right.$ $\left.\sigma\left(\mu F T x_{t}-\gamma V x_{t}\right)\right)$. Assume that $\left\{t_{n}\right\} \subset(0,1)$ is a sequence such that $t_{n} \rightarrow 0$ as $n \rightarrow \infty$. Put $x_{n}:=x_{t_{n}}$ and $y_{n}:=y_{t_{n}}$. For $z \in \operatorname{Fix}(T)$, we note that

$$
\begin{aligned}
x_{n}-z= & Q_{C} y_{n}-y_{n}+y_{n}-z \\
= & Q_{C} y_{n}-y_{n}+\left(I-t_{n} G\right)\left(T x_{n}-z\right)+t_{n}\left(T x_{n}-\sigma\left(\mu F T x_{n}-\gamma V x_{n}\right)-G z\right) \\
= & Q_{C} y_{n}-y_{n}+\left(I-t_{n} G\right)\left(T x_{n}-z\right)+t_{n}\left[(I-\sigma \mu F) T x_{n}+\sigma \gamma V x_{n}-G z\right] \\
= & Q_{C} y_{n}-y_{n}+\left(I-t_{n} G\right)\left(T x_{n}-z\right)+t_{n}\left[(I-\sigma \mu F)\left(T x_{n}-z\right)+\sigma \gamma\left(V x_{n}-V z\right)\right] \\
& +t_{n}[(I-\sigma \mu F) z+\sigma \gamma V z-G z] .
\end{aligned}
$$

By Lemma 2.4, we have

$$
\left\langle Q_{C} y_{n}-y_{n}, j_{q}\left(Q_{C} y_{n}-z\right)\right\rangle \leq 0 \text {. }
$$


It follows from (3.9) and (3.10) that

$$
\begin{aligned}
\left\|x_{n}-z\right\|^{q}= & \left\langle Q_{C} y_{n}-y_{n}, j_{q}\left(Q_{C} y_{n}-z\right)\right\rangle+\left\langle y_{n}-z, j_{q}\left(x_{n}-z\right)\right\rangle \\
\leq & \left\langle\left(I-t_{n} G\right)\left(T x_{n}-z\right), j_{q}\left(x_{n}-z\right)\right\rangle+t_{n}\left\langle(I-\sigma \mu F)\left(T x_{n}-z\right), j_{q}\left(x_{n}-z\right)\right\rangle \\
& +t_{n} \sigma \gamma\left\langle V x_{n}-V z, j_{q}\left(x_{n}-z\right)\right\rangle+t_{n}\left\langle(I-\sigma \mu F) z+\sigma \gamma V z-G z, j_{q}\left(x_{n}-z\right)\right\rangle \\
\leq & {\left[1-t_{n}\left(\sigma(\tau-\gamma L)-L_{\delta, \lambda}\right)\right]\left\|x_{n}-z\right\|^{q} } \\
& +t_{n}\left\langle(I-\sigma \mu F) z+\sigma \gamma V z-G z, j_{q}\left(x_{n}-z\right)\right\rangle,
\end{aligned}
$$

which implies that

$$
\left\|x_{n}-z\right\|^{q} \leq \frac{1}{\sigma(\tau-\gamma L)-L_{\delta, \lambda}}\left\langle(I-\sigma \mu F) z+\sigma \gamma V z-G z, j_{q}\left(x_{n}-z\right)\right\rangle .
$$

In particular, we have

$$
\left\|x_{n_{i}}-z\right\|^{q} \leq \frac{1}{\sigma(\tau-\gamma L)-L_{\delta, \lambda}}\left\langle(I-\sigma \mu F) z+\sigma \gamma V z-G z, j_{q}\left(x_{n_{i}}-z\right)\right\rangle .
$$

By reflexivity of a Banach space $X$ and boundedness of $\left\{x_{n}\right\}$, there exists a subsequence $\left\{x_{n_{i}}\right\}$ of $\left\{x_{n}\right\}$ such that $x_{n_{i}} \rightarrow \tilde{x}$ as $i \rightarrow \infty$. Since a Banach space $X$ has a weakly sequentially continuous generalized duality mapping and by (3.11), we obtain $x_{n_{i}} \rightarrow \tilde{x}$. By Proposition 3.3(ii), we have $x_{n_{i}}-T x_{n_{i}} \rightarrow 0$ as $i \rightarrow \infty$. Hence, it follows from Lemma 2.12 that $\tilde{x} \in \operatorname{Fix}(T)$.

Next, we show that $\tilde{x}$ solves the variational inequality (3.3). We note that

$$
x_{t}=Q_{C} y_{t}=Q_{C} y_{t}-y_{t}+(I-t G) T x+t\left(T x_{t}-\sigma\left(\mu F T x_{t}-\gamma V x_{t}\right)\right) \text {, }
$$

we derive

$$
(G-I+\sigma(\mu F-\gamma V)) x_{t}=\frac{1}{t}\left(Q_{C} y_{t}-y_{t}\right)-\frac{1}{t}\left((I-t G)(I-T) x_{t}+t(I-\sigma \mu F)(I-T) x_{t}\right)
$$

Since $I-T$ is accretive (i.e., $\left\langle(I-T) x-(I-T) y, j_{q}(x-y)\right\rangle \geq 0$ for $\left.x, y \in C\right)$. For all $v \in \operatorname{Fix}(T)$, it follows from (3.10) and (3.12) that

$$
\begin{aligned}
\langle(G- & \left.I+\sigma(\mu F-\gamma V)) x_{t}, j_{q}\left(x_{t}-v\right)\right\rangle \\
= & \frac{1}{t}\left\langle Q_{C} y_{t}-y_{t}, j_{q}\left(Q_{C} y_{t}-v\right)\right\rangle-\frac{1}{t}\left\langle(I-t G)(I-T) x_{t}, j_{q}\left(x_{t}-v\right)\right\rangle \\
& \quad-\left\langle(I-\sigma \mu F)(I-T) x_{t}, j_{q}\left(x_{t}-v\right)\right\rangle \\
\leq & -\frac{1}{t}\left\langle(I-T) x_{t}-(I-T) v, j_{q}\left(x_{t}-v\right)\right\rangle+\left\langle G(I-T) x_{t}, j_{q}\left(x_{t}-v\right)\right\rangle \\
& \quad-\left\langle(I-T) x_{t}-(I-T) v, j_{q}\left(x_{t}-v\right)\right\rangle+\sigma \mu\left\langle F(I-T) x_{t}, j_{q}\left(x_{t}-v\right)\right\rangle \\
\leq & \left\langle G(I-T) x_{t}, j_{q}\left(x_{t}-v\right)\right\rangle+\sigma \mu\left\langle F(I-T) x_{t}, j_{q}\left(x_{t}-v\right)\right\rangle \\
\leq & \|G\|\left\|x_{t}-T x_{t}\right\|\left\|x_{t}-v\right\|^{q-1}+\sigma \mu\|F\|\left\|x_{t}-T x_{t}\right\|\left\|x_{t}-v\right\|^{q-1} \\
\leq & \left\|x_{t}-T x_{t}\right\| M_{1},
\end{aligned}
$$


where $M_{1}>0$ is an appropriate constant such that $M_{1}=\sup _{t \in(0,1)}\left\{\|G\|\left\|x_{t}-v\right\|^{q-1}, \sigma \mu\|F\| \times\right.$ $\left.\left\|x_{t}-v\right\|^{q-1}\right\}$. Now, replacing $t$ in (3.13) with $t_{n}$ and taking the limit as $n \rightarrow \infty$, we notice that $x_{t_{n}}-T x_{t_{n}} \rightarrow \tilde{x}-T \tilde{x}=0$, we obtain

$$
\left\langle(G-I+\sigma(\mu F-\gamma V)) \tilde{x}, j_{q}(\tilde{x}-v)\right\rangle \leq 0 .
$$

That is, $\tilde{x} \in \operatorname{Fix}(T)$ is the solution of the variational inequality (3.3). Consequently, $\tilde{x}=x^{*}$ by uniqueness. In a summary, we have shown that each cluster point of $\left\{x_{t}\right\}$ is equal to $x^{*}$. Therefore $x_{t} \rightarrow x^{*}$ as $t \rightarrow 0$. This completes the proof.

\subsection{Explicit iteration scheme}

Theorem 3.5 Let C be a nonempty, closed, and convex subset of a real q-uniformly smooth and uniformly convex Banach space $X$ which admits a weakly sequentially continuous generalized duality mapping $j_{q}$. Let $Q_{C}$ be a sunny nonexpansive retraction such that $X$ onto $C$. Let $F: C \rightarrow X$ be a $\kappa$-Lipschitzian and $\eta$-strongly accretive operator with constants $\kappa, \eta>0, G: C \rightarrow X$ be a $\delta$-strongly accretive and $\lambda$-strictly pseudo-contractive mapping with $\delta+\lambda>1, V: C \rightarrow X$ be an L-Lipschitzian mapping with constant $L \geq 0$. Let $\left\{S_{i}\right\}_{i=1}^{\infty}$ be an infinite family of $\lambda_{i}$-strictly pseudo-contractive mapping from $C$ into itself such that $\mathcal{F}:=\bigcap_{i=1}^{\infty} \operatorname{Fix}\left(S_{i}\right) \neq \emptyset$. For given $x_{1} \in C$, define the sequence $\left\{x_{n}\right\}$ by

$$
x_{n+1}=Q_{C}\left[\left(I-\alpha_{n} G\right) W_{n} x_{n}+\alpha_{n}\left(W_{n} x_{n}-\sigma\left(\mu F W_{n} x_{n}-\gamma V x_{n}\right)\right)\right], \quad \forall n \geq 1,
$$

where $\left\{\alpha_{n}\right\}$ is a sequence in $(0,1)$ which satisfies the following conditions:

(C1) $\lim _{n \rightarrow \infty} \alpha_{n}=0$ and $\sum_{n=1}^{\infty} \alpha_{n}=\infty$;

(C2) $\left|\alpha_{n+1}-\alpha_{n}\right| \leq \circ\left(\alpha_{n}\right)+\sigma_{n}$ with $\sum_{n=1}^{\infty} \sigma_{n}<\infty$.

Suppose in addition that $\left\{\theta_{n, k}\right\}$ satisfies (H1)-(H3). Then the sequence $\left\{x_{n}\right\}$ defined by (3.14) converges strongly to $x^{*} \in \mathcal{F}$ as $n \rightarrow \infty$, where $x^{*}$ is the unique solution of the variational inequality

$$
\left\langle(G-I+\sigma(\mu F-\gamma V)) x^{*}, j_{q}\left(x^{*}-v\right)\right\rangle \leq 0, \quad \forall v \in \mathcal{F} .
$$

Proof From the condition (C1), we may assume, without loss of generality, that $\alpha_{n} \leq$ $\min \left\{1, \frac{1}{q \tau}\right\}$ for all $n \in \mathbb{N}$. First, we show that $\left\{x_{n}\right\}$ is bounded. Take $p \in \mathcal{F}$, and denote a mapping $S_{n}^{\alpha_{n}}: C \rightarrow C$ by

$$
S_{n}^{\alpha_{n}} x:=Q_{C}\left[\left(I-\alpha_{n} G\right) W_{n} x+\alpha_{n}\left(W_{n} x-\sigma\left(\mu F W_{n} x-\gamma V x\right)\right)\right], \quad \forall x \in C .
$$

Then we have

$$
S_{n}^{\alpha_{n}} p=Q_{C}\left[\left(I-\alpha_{n} G\right) W_{n} p+\alpha_{n}\left(W_{n} p-\sigma\left(\mu F W_{n} p-\gamma V p\right)\right)\right] .
$$

From (3.1), we have

$$
\begin{aligned}
\left\|x_{n+1}-p\right\| & \leq\left\|S_{n}^{\alpha_{n}} x_{n}-S_{n}^{\alpha_{n}} p\right\|+\left\|S_{n}^{\alpha_{n}} p-p\right\| \\
& \leq\left(1-\alpha_{n} \theta\right)\left\|x_{n}-p\right\|+\left\|Q_{C}\left[\left(I-\alpha_{n} G\right) p+\alpha_{n}(p-\sigma(\mu F p-\gamma V p))\right]-Q_{C} p\right\|
\end{aligned}
$$




$$
\begin{aligned}
& \leq\left(1-\alpha_{n} \theta\right)\left\|x_{n}-p\right\|+\alpha_{n}\|-G p+p-\sigma(\mu F p-\gamma V p)\| \\
& \leq\left(1-\alpha_{n} \theta\right)\left\|x_{n}-p\right\|+\alpha_{n}(\|I-G\|\|p\|+\sigma\|\mu F p-\gamma V p\|) \\
& \leq\left(1-\alpha_{n} \theta\right)\left\|x_{n}-p\right\|+\alpha_{n} \theta \frac{\|I-G\|\|p\|+\sigma \mu\|F p\|+\gamma\|V p\|}{\theta} \\
& \leq \max \left\{\left\|x_{n}-p\right\|, \frac{\|I-G\|\|p\|+\sigma \mu\|F p\|+\gamma\|V p\|}{\theta}\right\},
\end{aligned}
$$

where $\theta:=\sigma(\tau-\gamma L)-L_{\delta, \lambda}$. By induction, we obtain

$$
\left\|x_{n}-p\right\| \leq \max \left\{\left\|x_{1}-p\right\|, \frac{\|I-G\|\|p\|+\sigma \mu\|F p\|+\gamma\|V p\|}{\theta}\right\}, \quad \forall n \geq 1
$$

Hence, $\left\{x_{n}\right\}$ is bounded, so are $\left\{V x_{n}\right\},\left\{F W_{n} x_{n}\right\}$, and $\left\{G W_{n} x_{n}\right\}$.

Next, we show that $\left\|x_{n+1}-x_{n}\right\| \rightarrow 0$ as $n \rightarrow \infty$. Set $S_{n}^{\alpha_{n}} x_{n}=Q_{C} y_{n}$, where $y_{n}=(I-$ $\left.\alpha_{n} G\right) W_{n} x_{n}+\alpha_{n}\left(W_{n} x_{n}-\sigma\left(\mu F W_{n} x_{n}-\gamma V x_{n}\right)\right)$. From (2.5), we have

$$
\begin{aligned}
\| & W_{n+1} x_{n}-W_{n} x_{n} \| \\
& =\left\|t_{1} T_{n+1,1} U_{n+1,2} x_{n}+\left(1-t_{1}\right) x_{n}-t_{1} T_{n, 1} U_{n, 2}-\left(1-t_{1}\right) x_{n}\right\| \\
& t_{1}\left\|T_{n+1,1} U_{n+1,2} x_{n}-T_{n, 1} U_{n, 2} x_{n}\right\| \\
& =t_{1}\left\|\left(\theta_{n+1,1} S_{1}+\left(1-\theta_{n+1,1}\right)\right) U_{n+1,2} x_{n}-T_{n, 1} U_{n, 2} x_{n}\right\| \\
& =t_{1}\left\|\left(\theta_{n, 1} S_{1}+\left(1-\theta_{n, 1}\right)\right) U_{n+1,2} x_{n}-T_{n, 1} U_{n, 2} x_{n}+\left(\theta_{n+1,1}-\theta_{n, 1}\right)\left(S_{1} U_{n+1,2} x_{n}-U_{n+1,2} x_{n}\right)\right\| \\
\leq & t_{1}\left\|T_{n, 1} U_{n+1,2} x_{n}-T_{n, 1} U_{n, 2} x_{n}\right\|+t_{1}\left|\theta_{n+1,2}-\theta_{n, 1}\right|\left\|S_{1} U_{n+1,2} x_{n}-U_{n+1,2} x_{n}\right\| \\
\leq & t_{1}\left\|T_{n, 1} U_{n+1,2} x_{n}-T_{n, 1} U_{n, 2} x_{n}\right\|+t_{1}\left|\theta_{n+1,2}-\theta_{n, 1}\right| M^{*} \\
\leq & t_{1}\left\|T_{n, 1} U_{n+1,2} x_{n}-T_{n, 1} U_{n, 2} x_{n}\right\|+t_{1} a_{n} M_{1} \\
& \vdots \\
\leq & \prod_{i=1}^{n} t_{i}\left\|U_{n+1, n+1} x_{n}-U_{n, n+1} x_{n}\right\|+\left(a_{n} \sum_{j=1}^{n} \sum_{i=1}^{j} t_{i}\right) M_{1} \\
\leq & \left(b^{n+1}+\frac{b}{1-b} a_{n}\right) M_{1}, \\
\leq & \prod_{i=1}^{n} t_{i}\left\|t_{n+1} T_{n+1, n+1} x_{n}+\left(1-t_{n+1}\right) x_{n}-x_{n}\right\|+\frac{b}{1-b} a_{n} M_{1} \\
\leq & \prod_{i=1}^{n+1} t_{i}\left\|T_{n+1, n+1} x_{n}-x_{n}\right\|+\frac{b}{1-b} a_{n} M_{1} \\
& (3.16)
\end{aligned}
$$

where $M_{1}=\inf _{i=1,2, \ldots}\left(\frac{1+2 \lambda_{i}^{q-1}}{\lambda_{i}^{q-1}}\right) \sup _{n \geq 1}\left\{\left\|x_{n}-p\right\|\right\}$ with $p \in \mathcal{F}$.

On the other hand, we note that

$$
\begin{aligned}
y_{n+1}-y_{n}= & \left(I-\alpha_{n+1} G\right) W_{n+1} x_{n}+\alpha_{n+1}\left[W_{n+1} x_{n}-\sigma\left(\mu F W_{n+1} x_{n}-\gamma V x_{n}\right)\right] \\
& -\left(I-\alpha_{n} G\right) W_{n} x_{n}-\alpha_{n}\left[W_{n} x_{n}-\sigma\left(\mu F W_{n} x_{n}-\gamma V x_{n}\right)\right]
\end{aligned}
$$




$$
\begin{aligned}
= & \left(I-\alpha_{n+1} G\right)\left(W_{n+1} x_{n}-W_{n} x_{n}\right)+\left(\alpha_{n}-\alpha_{n+1}\right) G W_{n} x_{n}+\left(\alpha_{n+1}-\alpha_{n}\right) W_{n+1} x_{n} \\
& +\alpha_{n}\left(W_{n+1} x_{n}-W_{n} x_{n}\right)+\sigma\left(\alpha_{n}-\alpha_{n+1}\right)\left(\mu F W_{n+1} x_{n}-\gamma V x_{n}\right) \\
& -\sigma \alpha_{n}\left[\mu F W_{n+1} x_{n}-\gamma V x_{n}-\left(\mu F W_{n} x_{n}-\gamma V x_{n}\right)\right] \\
= & {\left[\left(1+\alpha_{n}\right) I-\alpha_{n+1} G\right]\left(W_{n+1} x_{n}-W_{n} x_{n}\right)+\left(\alpha_{n+1}-\alpha_{n}\right)\left[W_{n+1} x_{n}-G W_{n} x_{n}\right] } \\
& +\sigma\left(\alpha_{n}-\alpha_{n+1}\right)\left[\mu F W_{n+1} x_{n}-\gamma V x_{n}\right]-\sigma \alpha_{n} \mu F\left[W_{n+1} x_{n}-W_{n} x_{n}\right] \\
= & {\left[\left(1+\alpha_{n}\right) I-\alpha_{n+1} G-\sigma \alpha_{n} \mu F\right]\left(W_{n+1} x_{n}-W_{n} x_{n}\right) } \\
& +\left(\alpha_{n+1}-\alpha_{n}\right)\left[W_{n+1} x_{n}-G W_{n} x_{n}\right]+\sigma\left(\alpha_{n}-\alpha_{n+1}\right)\left[\mu F W_{n+1} x_{n}-\gamma V x_{n}\right] .
\end{aligned}
$$

Hence, we have

$$
\begin{aligned}
\left\|S_{n+1}^{\alpha_{n+1}} x_{n}-S_{n}^{\alpha_{n}} x_{n}\right\|= & \left\|Q_{C} y_{n+1}-Q_{C} y_{n}\right\| \\
\leq & \left\|y_{n+1}-y_{n}\right\| \\
\leq & \left\|\left(1+\alpha_{n}\right) I-\alpha_{n+1} G-\sigma \alpha_{n} \mu F\right\|\left\|W_{n+1} x_{n}-W_{n} x_{n}\right\| \\
& +\left|\alpha_{n+1}-\alpha_{n}\right|\left\|W_{n+1} x_{n}-G W_{n} x_{n}\right\| \\
& +\sigma\left|\alpha_{n+1}-\alpha_{n}\right|\left\|\mu F W_{n+1} x_{n}-\gamma V x_{n}\right\| \\
\leq & \left(\left\|W_{n+1} x_{n}-W_{n} x_{n}\right\|+\left|\alpha_{n+1}-\alpha_{n}\right|\right) M_{2},
\end{aligned}
$$

where $M_{2}=\sup _{n \geq 1}\left\{\left\|\left(1+\alpha_{n}\right) I-\alpha_{n+1} G-\sigma \alpha_{n} \mu F\right\|,\left\|W_{n+1} x_{n}-G W_{n} x_{n}\right\|, \sigma \| \mu F W_{n+1} x_{n}-\right.$ $\left.\gamma V x_{n} \|\right\}$. It follows from (3.1) and (3.16) that

$$
\begin{aligned}
\left\|x_{n+2}-x_{n+1}\right\| & \leq\left\|S_{n+1}^{\alpha_{n+1}} x_{n+1}-S_{n+1}^{\alpha_{n+1}} x_{n}\right\|+\left\|S_{n+1}^{\alpha_{n+1}} x_{n}-S_{n}^{\alpha_{n}} x_{n}\right\| \\
& \leq\left(1-\alpha_{n+1} \theta\right)\left\|x_{n+1}-x_{n}\right\|+\left(\left|\alpha_{n+1}-\alpha_{n}\right|+\left\|W_{n+1} x_{n}-W_{n} x_{n}\right\|\right) M_{2} \\
& \leq\left(1-\alpha_{n+1} \theta\right)\left\|x_{n+1}-x_{n}\right\|+\left(\circ\left(\alpha_{n}\right)+\sigma_{n}\right) M_{2}+\left\|W_{n+1} x_{n}-W_{n} x_{n}\right\| M_{2} \\
& \leq\left(1-\alpha_{n+1} \theta\right)\left\|x_{n+1}-x_{n}\right\|+\circ\left(\alpha_{n}\right) M_{2}+\left(\sigma_{n}+b^{n+1}+\frac{b}{1-b} a_{n}\right) M,
\end{aligned}
$$

where $M=\max \left\{M_{1}, M_{2}\right\}$. Then, by Lemma 2.13, we have

$$
\lim _{n \rightarrow \infty}\left\|x_{n+1}-x_{n}\right\|=0 .
$$

Next, we show that $\lim _{n \rightarrow \infty}\left\|x_{n}-W x_{n}\right\|=0$. Since

$$
\begin{aligned}
\left\|x_{n}-W_{n} x_{n}\right\| \leq & \left\|x_{n}-x_{n+1}\right\|+\left\|x_{n+1}-W_{n} x_{n}\right\| \\
= & \left\|x_{n}-x_{n+1}\right\|+\| Q_{C}\left[\left(I-\alpha_{n} G\right) W_{n} x_{n}+\alpha_{n}\left(W_{n} x_{n}-\sigma\left(\mu F W_{n} x_{n}-\gamma V x_{n}\right)\right)\right] \\
& \quad-Q_{C} W_{n} x_{n} \| \\
\leq & \left\|x_{n}-x_{n+1}\right\|+\alpha_{n}\left\|(I-G) W_{n} x_{n}-\sigma\left(\mu F W_{n} x_{n}-\gamma V x_{n}\right)\right\| .
\end{aligned}
$$

From (3.18) and the condition (C1), we obtain

$$
\lim _{n \rightarrow \infty}\left\|x_{n}-W_{n} x_{n}\right\|=0 .
$$


At the same time, observe that

$$
\left\|x_{n}-W x_{n}\right\| \leq\left\|x_{n}-W_{n} x_{n}\right\|+\left\|W_{n} x_{n}-W x_{n}\right\| .
$$

It follows from (3.19) and Lemma 2.11, we have

$$
\lim _{n \rightarrow \infty}\left\|x_{n}-W x_{n}\right\|=0
$$

Next, we show that

$$
\limsup _{n \rightarrow \infty}\left\langle[I-G+\sigma(\gamma V-\mu F)] x^{*}, j_{q}\left(x_{n}-x^{*}\right)\right\rangle \leq 0
$$

where $x^{*}$ is the same as in Theorem 3.4. Since $\left\{x_{n}\right\}$ is bounded, there exists a subsequence $\left\{x_{n_{i}}\right\}$ of $\left\{x_{n}\right\}$ such that

$$
\begin{aligned}
& \underset{n \rightarrow \infty}{\limsup }\left\langle[I-G+\sigma(\gamma V-\mu F)] x^{*}, j_{q}\left(x_{n}-x^{*}\right)\right\rangle \\
& \quad=\lim _{i \rightarrow \infty}\left\langle[I-G+\sigma(\gamma V-\mu F)] x^{*}, j_{q}\left(x_{n_{i}}-x^{*}\right)\right\rangle .
\end{aligned}
$$

By reflexivity of a Banach space $X$ and boundedness of $\left\{x_{n}\right\}$, without loss of generality, we may assume that $x_{n_{i}} \rightarrow v$ as $i \rightarrow \infty$. It follows from (3.20) and Lemma 2.12 that $v \in \mathcal{F}$. Since a Banach space $X$ has a weakly sequentially continuous generalized duality mapping, we obtain

$$
\begin{aligned}
& \limsup _{n \rightarrow \infty}\left\langle[I-G+\sigma(\gamma V-\mu F)] x^{*}, j_{q}\left(x_{n}-x^{*}\right)\right\rangle \\
& \quad=\lim _{i \rightarrow \infty}\left\langle[I-G+\sigma(\gamma V-\mu F)] x^{*}, j_{q}\left(x_{n_{i}}-x^{*}\right)\right\rangle \\
& \quad=\left\langle[I-G+\sigma(\gamma V-\mu F)] x^{*} j_{q}\left(v-x^{*}\right)\right\rangle \leq 0 .
\end{aligned}
$$

Finally, we show that $x_{n} \rightarrow x^{*}$ as $n \rightarrow \infty$. Set $x_{n+1}=Q_{C} y_{n}$, where $y_{n}=\left(I-\alpha_{n} G\right) W_{n} x_{n}+$ $\alpha_{n}\left(W_{n} x_{n}-\sigma\left(\mu W_{n} x_{n}-\gamma V x_{n}\right)\right)$. From Lemmas 2.4 and 2.5 , we have

$$
\begin{aligned}
&\left\|x_{n+1}-x^{*}\right\|^{q} \\
&=\left\langle y_{n}-x^{*}, j_{q}\left(x_{n+1}-x^{*}\right)\right\rangle+\left\langle Q_{C} y_{n}-y_{n}, j_{q}\left(x_{n+1}-x^{*}\right)\right\rangle \\
& \leq\left\langle y_{n}-x^{*}, j_{q}\left(x_{n+1}-x^{*}\right)\right\rangle \\
&=\left\langle\left(I-\alpha_{n} G\right)\left(W_{n} x_{n}-x^{*}\right), j_{q}\left(x_{n+1}-x^{*}\right)\right\rangle+\alpha_{n}\left\langle(I-\sigma \mu F)\left(W_{n} x_{n}-x^{*}\right), j_{q}\left(x_{n+1}-x^{*}\right)\right\rangle \\
& \quad+\alpha_{n} \sigma \gamma\left\langle V x_{n}-V x^{*}, j_{q}\left(x_{n+1}-x^{*}\right)\right\rangle+\alpha_{n}\left\langle(I-\sigma \mu F) x^{*}+\sigma \gamma V x^{*}-G x^{*}, j_{q}\left(x_{n+1}-x^{*}\right)\right\rangle \\
& \leq\left(1-\alpha_{n}\left(1-L_{\delta, \lambda}\right)\right)\left\|x_{n}-x^{*}\right\|\left\|x_{n+1}-x^{*}\right\|^{q-1}+\alpha_{n}(1-\sigma \tau)\left\|x_{n}-x^{*}\right\|\left\|x_{n+1}-x^{*}\right\|^{q-1} \\
&+\alpha_{n} \sigma \gamma L\left\|x_{n}-x^{*}\right\|\left\|x_{n+1}-x^{*}\right\|^{q-1}+\alpha_{n}\left\langle x^{*}-G x^{*}+\sigma\left(\gamma V x^{*}-\mu F x^{*}\right), j_{q}\left(x_{n+1}-x^{*}\right)\right\rangle \\
&=\left(1-\alpha_{n}\left(\sigma(\tau-\gamma L)-L_{\delta, \lambda}\right)\right)\left\|x_{n}-x^{*}\right\|\left\|x_{n+1}-x^{*}\right\|^{q-1} \\
&+\alpha_{n}\left\langle x^{*}-G x^{*}+\sigma\left(\gamma V x^{*}-\mu F x^{*}\right), j_{q}\left(x_{n+1}-x^{*}\right)\right\rangle
\end{aligned}
$$




$$
\begin{aligned}
\leq & \left(1-\alpha_{n}\left(\sigma(\tau-\gamma L)-L_{\delta, \lambda}\right)\right)\left[\frac{1}{q}\left\|x_{n}-x^{*}\right\|^{q}+\left(\frac{q-1}{q}\right)\left\|x_{n+1}-x^{*}\right\|^{q}\right] \\
& +\alpha_{n}\left(x^{*}-G x^{*}+\sigma\left(\gamma V x^{*}-\mu F x^{*}\right), j_{q}\left(x_{n+1}-x^{*}\right)\right\rangle,
\end{aligned}
$$

which implies that

$$
\begin{aligned}
\left\|x_{n+1}-x^{*}\right\|^{q} \leq & \left(1-\alpha_{n}\left(\sigma(\tau-\gamma L)-L_{\delta, \lambda}\right)\right)\left\|x_{n}-x^{*}\right\|^{q} \\
& +\frac{q \alpha_{n}}{1+(q-1)\left(\sigma(\tau-\gamma L)-L_{\delta, \lambda}\right)} \\
& \times\left\langle x^{*}-G x^{*}+\sigma\left(\gamma V x^{*}-\mu F x^{*}\right), j_{q}\left(x_{n+1}-x^{*}\right)\right\rangle .
\end{aligned}
$$

We can write (3.22) to the formula

$$
\left\|x_{n+1}-x^{*}\right\|^{q} \leq\left(1-\tau_{n}\right)\left\|x_{n}-x\right\|^{q}+\xi_{n}
$$

where $\tau_{n}:=\left(\sigma(\tau-\gamma L)-L_{\delta, \lambda}\right) \alpha_{n}$ and $\xi_{n}:=\frac{q \alpha_{n}}{1+(q-1)\left(\sigma(\tau-\gamma L)-L_{\delta, \lambda}\right)}\left\langle x^{*}-G x^{*}+\sigma\left(\gamma V x^{*}-\mu F x^{*}\right)\right.$, $\left.j_{q}\left(x_{n+1}-x^{*}\right)\right\rangle$. Put $c_{n}=\max \left\{0, \xi_{n}\right\}$, from (3.21), we have $c_{n} \rightarrow 0$ as $n \rightarrow \infty$. Then we can rewrite (3.23) as

$$
\begin{aligned}
\left\|x_{n+1}-x^{*}\right\|^{q} & \leq\left(1-\tau_{n}\right)\left\|x_{n}-x^{*}\right\|^{q}+c_{n} \\
& \leq\left(1-\tau_{n}\right)\left\|x_{n}-x^{*}\right\|^{q}+o\left(\alpha_{n}\right) .
\end{aligned}
$$

Therefore, by Lemma 2.13, we conclude that $x_{n} \rightarrow x^{*}$ as $n \rightarrow \infty$. This completes the proof.

\section{Some applications}

In this section, we will utilize Theorems 3.4 and 3.5 to study some strong convergence theorems in $L_{p}$ (or $\ell_{p}$ ) spaces with $1<p<\infty$. It well known that Hilbert spaces, $L_{p}$ (or $\ell_{p}$ ) spaces with $1<p<\infty$ and the Sobolev spaces $W_{m}^{p}$ with $1<p<\infty$ are $q$-uniformly smooth, i.e.,

$$
L_{p}\left(\text { or } \ell_{p}\right) \text { or } W_{m}^{p} \text { is } \begin{cases}2 \text {-uniformly smooth, } & \text { if } 2 \leq p<\infty \\ p \text {-uniformly smooth, } & \text { if } 1<p \leq 2 .\end{cases}
$$

Furthermore, we have the following properties of $L_{p}$ (or $\ell_{p}$ ) spaces with $1<p<\infty$ (see [36, 39]):

(1) For $2 \leq p<\infty$, the spaces $L_{p}$ (or $\ell_{p}$ ) are 2-uniformly smooth with $C_{q}=C_{2}=p-1$.

(2) For $1<p \leq 2$, the spaces $L_{p}$ (or $\ell_{p}$ ) are $p$-uniformly smooth with $C_{q}=C_{p}=\left(1+t_{p}^{p-1}\right)\left(1+t_{p}\right)^{1-p}$, where $t_{p}$ is the unique solution of the equation

$$
(p-2) t^{p-1}+(p-1) t^{p-2}-1=0, \quad 0<t<1 .
$$

(3) Every Hilbert spaces are 2-uniformly smooth with $C_{q}=C_{2}=1$.

(4) Every $L_{p}$ (or $\ell_{p}$ ) spaces with $1<p<\infty$ are $q$-uniformly smooth and uniformly convex. 
(5) Every $\ell_{p}$ spaces with $1<p<\infty$ have weakly sequentially continuous generalized duality mappings, but $L_{p}$ spaces $(1<p<\infty, p \neq 2)$ do not have weakly sequentially continuous generalized duality mappings.

Lemma 4.1 Let $X:=L_{p}\left(\right.$ or $\left.\ell_{p}\right)$ with $1<p \leq 2$. Let $C$ be a nonempty, closed, and convex subset of $X$. Let $F: C \rightarrow X$ be a $\kappa$-Lipschitzian and $\eta$-strongly accretive operator with constants $\kappa, \eta>0$. Let $0<\mu<\left(\frac{p \eta}{D_{p} \kappa^{p}}\right)^{\frac{1}{p-1}}$ and $\tau=\mu\left(\eta-\frac{D_{p} \mu^{p-1} \kappa^{p}}{p}\right)$. Then for $t \in\left(0, \min \left\{1, \frac{1}{p \tau}\right\}\right)$, the mapping $S: C \rightarrow X$ defined by $S:=I-t \mu F$ is a contraction with constant $1-t \tau$.

Lemma 4.2 Let $X:=L_{p}\left(\right.$ or $\left.\ell_{p}\right)$ with $2 \leq p<\infty$. Let $C$ be a nonempty, closed, and convex subset of $X$. Let $F: C \rightarrow X$ be a $\kappa$-Lipschitzian and $\eta$-strongly accretive operator with constants $\kappa, \eta>0$. Let $0<\mu<\frac{2 \eta}{(p-1) \kappa^{2}}$ and $\tau=\mu\left(\eta-\frac{(p-1) \mu \kappa^{2}}{2}\right)$. Then for $t \in\left(0, \min \left\{1, \frac{1}{2 \tau}\right\}\right)$, the mapping $S: C \rightarrow X$ defined by $S:=I-t \mu F$ is a contraction with constant $1-t \tau$.

Lemma 4.3 Let $X:=H$ be a real Hilbert space. Let $C$ be a nonempty, closed, and convex subset of $X$. Let $F: C \rightarrow X$ be a $\kappa$-Lipschitzian and $\eta$-strongly accretive operator with constants $\kappa, \eta>0$. Let $0<\mu<\frac{2 \eta}{\kappa^{2}}$ and $\tau=\mu\left(\eta-\frac{\mu \kappa^{2}}{2}\right)$. Then for $t \in\left(0, \min \left\{1, \frac{1}{2 \tau}\right\}\right)$, the mapping $S: C \rightarrow X$ defined by $S:=I-t \mu F$ is a contraction with constant $1-t \tau$.

\subsection{Implicit iteration schemes}

Theorem 4.4 Let $C$ be a nonempty, closed, and convex subset of an $\ell_{p}$ space for $1<p \leq 2$. Let $Q_{C}, F, G, V$, and $T$ be the same as in Theorem 3.4. Assume that $0<\mu<\left(\frac{p \eta}{D_{p} k^{p}}\right)^{\frac{1}{p-1}}$ and $0 \leq \gamma L<\tau$, where $\tau=\mu\left(\eta-\frac{D_{p} \mu^{p-1} \kappa^{p}}{p}\right)$. For $\sigma \in\left(\frac{L_{\delta, \lambda}}{\tau-\gamma L}, \min \left\{1, \frac{1}{p \tau}, \frac{1+L_{\delta, \lambda}}{\tau-\gamma L}\right\}\right)$ and $t \in(0,1)$, the sequence $\left\{x_{t}\right\}$ defined by (3.2) converges strongly to $x^{*} \in \operatorname{Fix}(T)$ as $t \rightarrow 0$, where $x^{*}$ is the unique solution of the variational inequality (3.3).

Theorem 4.5 Let $C$ be a nonempty, closed, and convex subset of an $\ell_{p}$ space for $2 \leq p<\infty$. Let $Q_{C}, F, G, V$, and $T$ be the same as in Theorem 3.4. Assume that $0<\mu<\frac{2 \eta}{(p-1) \kappa^{2}}$ and $0 \leq \gamma L<\tau$, where $\tau=\mu\left(\eta-\frac{(p-1) \mu \kappa^{2}}{2}\right)$. For $\sigma \in\left(\frac{L_{\delta, \lambda}}{\tau-\gamma L}, \min \left\{1, \frac{1}{2 \tau}, \frac{1+L_{\delta, \lambda}}{\tau-\gamma L}\right\}\right)$ and $t \in(0,1)$, the sequence $\left\{x_{t}\right\}$ defined by (3.2) converges strongly to $x^{*} \in \operatorname{Fix}(T)$ as $t \rightarrow 0$, where $x^{*}$ is the unique solution of the variational inequality (3.3).

Remark 4.6 If the spaces $L_{p}$ has a weakly sequentially continuous generalized duality mappings, then we obtain Theorems 4.4 and 4.5 hold for $L_{p}$ spaces with $1<p<\infty, p \neq 2$.

\subsection{Explicit iteration schemes}

Theorem 4.7 Let $C$ be a nonempty, closed, and convex subset of an $\ell_{p}$ space for $1<p \leq 2$. Let $Q_{C}, F, G, V$, and $W_{n}$ be the same as in Theorem 3.5. Let $\left\{\alpha_{n}\right\}$ and $\left\{\beta_{n}\right\}$ are sequences in $(0,1)$ which satisfy the conditions $(\mathrm{C} 1)$ and $(\mathrm{C} 2)$ in Theorem 3.5 and $\left\{\theta_{n, k}\right\}$ satisfies $(\mathrm{H} 1)$ (H3). Then the sequence $\left\{x_{n}\right\}$ defined by (3.14) converges strongly to $x^{*} \in \mathcal{F}$ as $n \rightarrow \infty$, where $x^{*}$ is the unique solution of the variational inequality (3.15).

Theorem 4.8 Let $C$ be a nonempty, closed, and convex subset of an $\ell_{p}$ space for $2 \leq p<\infty$. Let $Q_{C}, F, G, V$, and $W_{n}$ be the same as in Theorem 3.5. Let $\left\{\alpha_{n}\right\}$ and $\left\{\beta_{n}\right\}$ are sequences in $(0,1)$ which satisfy the conditions $(\mathrm{C} 1)$ and $(\mathrm{C} 2)$ in Theorem 3.5 and $\left\{\theta_{n, k}\right\}$ satisfies (H1)(H3). Then the sequence $\left\{x_{n}\right\}$ defined by (3.14) converges strongly to $x^{*} \in \mathcal{F}$ as $n \rightarrow \infty$, where $x^{*}$ is the unique solution of the variational inequality (3.15). 
Remark 4.9 If the spaces $L_{p}$ has a weakly sequentially continuous generalized duality mappings, then we obtain Theorems 4.7 and 4.8 hold for $L_{p}$ spaces with $1<p<\infty, p \neq 2$.

\section{Numerical examples}

In this section, we give a simple example and some numerical experiment result to explain the convergence of the sequence (3.14) as follows:

Example 5.1 Let $X=\mathbb{R}$ and $C=\left[0, \frac{1}{2}\right]$. Let $q=2$ and $j_{q}=I$. We define a mapping $Q_{C}$ as follows:

$$
Q_{C} x= \begin{cases}\frac{x}{|x|}, & x \in(-\infty, 0) \cup\left(\frac{1}{2}, \infty\right), \\ x, & x \in\left[0, \frac{1}{2}\right] .\end{cases}
$$

In terms of Theorem 3.5, set $\sigma=\mu=\gamma=1$ and $\alpha_{n}=\frac{1}{n}$. Then we see that $\alpha_{n}=\frac{1}{n}$ satisfies (C1) and (C2) with $\sigma_{n}=\frac{1}{n^{2}}$. Moreover, we define the mappings $F, G$, and $V$ as follows:

$$
F x=\frac{1}{3}\left(x^{2}+2 x\right), \quad G x=x \text { and } \quad V x=x^{2} .
$$

It is easy to observe that $F$ is 1-Lipschitzian and $\frac{2}{3}$-strongly accretive, $G$ is 1 -strongly accretive and $\lambda$-strictly pseudo-contraction for $\lambda>0$ and $V$ is 1-Lipschitzian. For each $n \in \mathbb{N}$, set $S_{n}=I$. We show that $W_{n}=I$. Since $T_{n, k}=\theta_{n, k} S_{k}+\left(1-\theta_{n, k}\right) I$, where $S_{k}$ is a $\lambda_{k}$-strictly pseudo-contractive mapping and $\left\{\theta_{n, k}\right\}$ satisfies (H1)-(H3). It is observe that $T_{n, k}$ is a nonexpansive mapping. From (2.5), we have

$$
\begin{aligned}
W_{1} & =U_{1,1}=t_{1} T_{1,1} U_{1,2}+\left(1-t_{1}\right) I \\
W_{2} & =U_{2,1}=t_{1} T_{2,1} U_{2,2}+\left(1-t_{1}\right) I \\
& =t_{1} T_{2,1}\left(t_{2} T_{2,2} U_{2,3}+\left(1-t_{2}\right) I\right)+\left(1-t_{1}\right) I \\
& =t_{1} t_{2} T_{2,1} T_{2,2} U_{2,3}+t_{1}\left(1-t_{2}\right) T_{2,1}+\left(1-t_{1}\right) I, \\
W_{3} & =U_{3,1}=t_{1} T_{3,1} U_{3,2}+\left(1-t_{1}\right) I \\
& =t_{1} T_{3,1}\left(t_{2} T_{3,2} U_{3,3}+\left(1-t_{2}\right) I\right)+\left(1-t_{1}\right) I \\
& =t_{1} t_{2} T_{3,1} T_{3,2} U_{3,3}+t_{1}\left(1-t_{2}\right) T_{3,1}+\left(1-t_{1}\right) I \\
& =t_{1} t_{2} T_{3,1} T_{3,2}\left(t_{3} T_{3,3} U_{3,4}+\left(1-t_{3}\right) I\right)+t_{1}\left(1-t_{2}\right) T_{3,1}+\left(1-t_{1}\right) I \\
& =t_{1} t_{2} t_{3} T_{3,1} T_{3,2} T_{3,3}+t_{1} t_{2}\left(1-t_{3}\right) T_{3,1} T_{3,2}+t_{1}\left(1-t_{2}\right) T_{3,1}+\left(1-t_{1}\right) I
\end{aligned}
$$

and we compute (2.5) in a similar way to above, we obtain

$$
\begin{aligned}
W_{n}= & U_{n, 1} \\
= & t_{1} t_{2} \cdots t_{n} T_{n, 1} T_{n, 2} \cdots T_{n, n}+t_{1} t_{2} \cdots t_{n-1}\left(1-t_{n}\right) T_{n, 1} T_{n, 2} \cdots T_{n, n-1} \\
& +t_{1} t_{2} \cdots t_{n-2}\left(1-t_{n-1}\right) T_{n, 1} T_{n, 2} \cdots T_{n, n-2}+\cdots+t_{1}\left(1-t_{2}\right) T_{n, 1}+\left(1-t_{1}\right) I .
\end{aligned}
$$

Since $S_{n}=I$ and $t_{n}=\alpha$, for all $n \in \mathbb{N}$, we have

$$
W_{n}=\left[\alpha^{n}+\alpha^{n-1}(1-\alpha)+\cdots+\alpha(1-\alpha)+(1-\alpha)\right]=I .
$$


Under the above assumptions, (3.14) is simplified as follows:

$$
\left\{\begin{array}{l}
x_{1} \in C:=\left[0, \frac{1}{2}\right], \\
x_{n+1}=\left(1-\frac{2}{3 n}\right) x_{n}+\frac{2}{3 n} x_{n}^{2} .
\end{array}\right.
$$

Since the assumptions of Theorem 3.5 are satisfied in Example 5.1, the sequence (5.1) converges to $x^{*}=0$, which is the unique fixed point of $S_{n}$.

Next, we show the numerical results by using MATLAB 7.11.0. We presented numerical comparisons for two cases of iteration process with different initial values, which show the convergence of the sequence (5.1).

When we choose $x_{1}=0.05$ and $x_{1}=0.1$, we see that the iteration process of sequence $\left\{x_{n}\right\}$ converges to $x^{*}=0$ at $n=8,615$ and $n=28,946$, respectively, as shown in Table 1 and Figures 1 and 2.

Table 1 The value of sequence $\left\{x_{n}\right\}$ with iteration values $x_{1}=0.05$ and $x_{1}=0.1$

\begin{tabular}{rllll}
\hline Iteration step $(\boldsymbol{n})$ & Sequence value $\left(\boldsymbol{x}_{\boldsymbol{n}}\right)$ & Error & Sequence value $\left(\boldsymbol{x}_{\boldsymbol{n}}\right)$ & Error \\
\hline 1 & 0.0500 & $5 \times 10^{-2}$ & 0.1000 & $1 \times 10^{-1}$ \\
2 & 0.0183 & $1.83 \times 10^{-2}$ & 0.0400 & $4 \times 10^{-2}$ \\
3 & 0.0123 & $1.23 \times 10^{-2}$ & 0.0272 & $2.72 \times 10^{-2}$ \\
4 & 0.0096 & $9.6 \times 10^{-3}$ & 0.0213 & $2.13 \times 10^{-2}$ \\
5 & 0.0080 & $8 \times 10^{-3}$ & 0.0178 & $1.78 \times 10^{-2}$ \\
$\vdots$ & $\vdots$ & $\vdots$ & $\vdots$ & $\vdots$ \\
1,658 & 0.0002 & $2 \times 10^{-4}$ & 0.00321 & $3.21 \times 10^{-3}$ \\
$\vdots$ & $\vdots$ & $\vdots$ & $\vdots$ & $\vdots$ \\
5,570 & 0.0002 & $2 \times 10^{-4}$ & 0.00217 & $2.17 \times 10^{-3}$ \\
$\vdots$ & $\vdots$ & $\vdots$ & $\vdots$ & $\vdots$ \\
8,614 & 0.0001 & $1 \times 10^{-4}$ & 0.00184 & $1.84 \times 10^{-3}$ \\
8,615 & 0.0000 & $1 \times 10^{-4}$ & 0.00184 & $1.84 \times 10^{-3}$ \\
$\vdots$ & $\vdots$ & $\vdots$ & $\vdots$ & $\vdots$ \\
28,945 & 0.0000 & $1 \times 10^{-4}$ & 0.0001 & $1 \times 10^{-4}$ \\
28,946 & 0.0000 & $1 \times 10^{-4}$ & 0.0000 & 0 \\
\hline
\end{tabular}

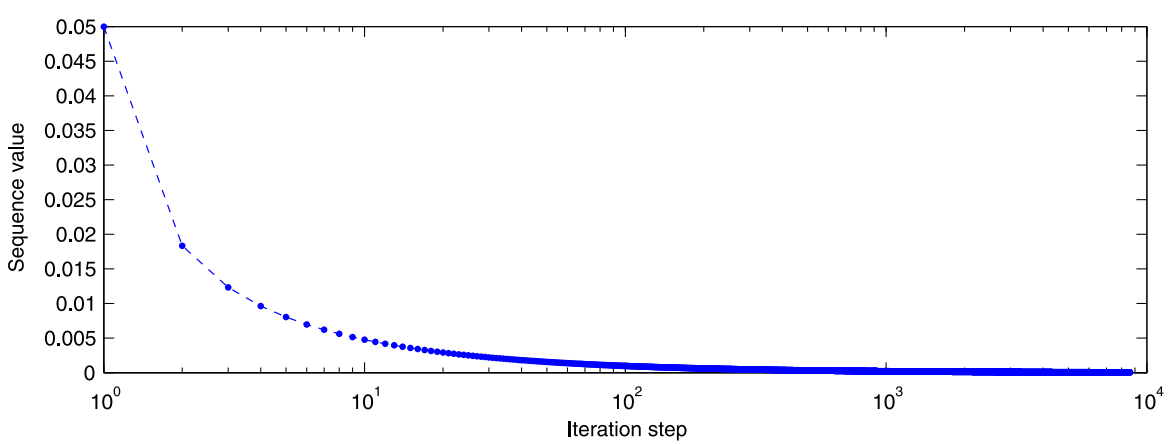

Figure 1 The iteration process with initial value $x_{1}=0.05$. 


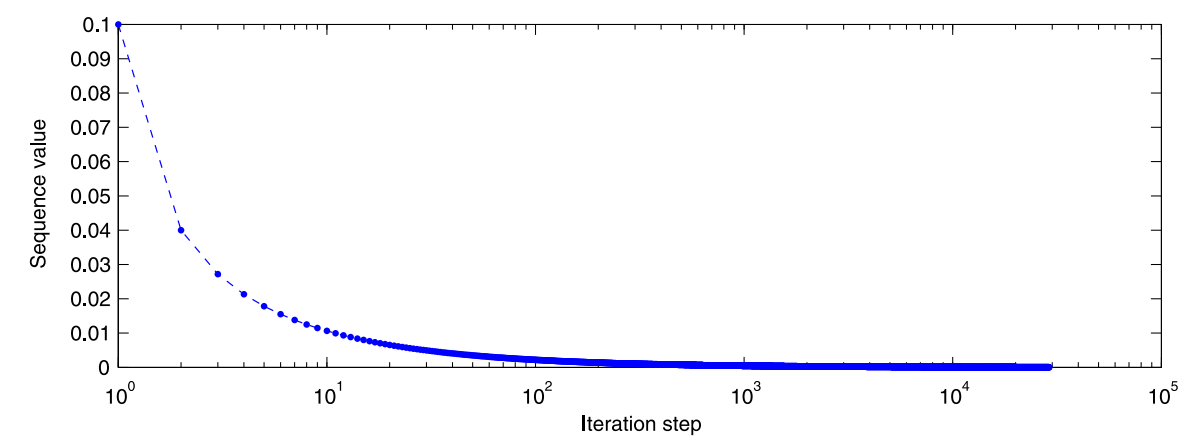

Figure 2 The iteration process with initial value $x_{1}=0.1$.

From the figures, we can see that $\left\{x_{n}\right\}$ is a monotone decreasing sequence and converges to 0 , but an iterative process with initial value $x_{1}=0.05$ is converges faster than an iterative process with initial value $x_{1}=0.1$.

Remark 5.2 Note that Lemma 3.1 and Lemma 3.2 play an important role in the proof of Theorems 3.4 and 3.5. These are proved in the framework of the more general $q$-uniformly smooth Banach space.

Remark 5.3 Our main result extends the main result of Ceng et al. [28] in the following respects:

(1) An iterative process (1.10) is to extend to a general iterative process defined over the set of fixed points of an infinite family of strict pseudo-contractions in a more general $q$-uniformly smooth Banach space.

(2) The self contraction mapping $f: H \rightarrow H$ in [28, Theorem 3.2] is extended to the case of a nonself Lipschitzian mapping $V: C \rightarrow X$ on a nonempty, closed, and convex subset $C$ of a real $q$-uniformly smooth Banach space $X$.

(3) The control condition (C3) in [28, Theorem 3.2] is removed by weaker than control condition $\left|\alpha_{n+1}-\alpha_{n}\right| \leq \circ\left(\alpha_{n}\right)+\sigma_{n}$ with $\sum_{n=1}^{\infty} \sigma_{n}<\infty$.

Furthermore, our method is extended to develop a new iterative method and method of proof is very different from that in Ceng et al. [28] because our method involves the sunny nonexpansive retraction and the infinite family of strict pseudo-contractions.

\section{Competing interests}

The authors declare that they have no competing interests.

\section{Authors' contributions}

All authors contributed equally and significantly in writing this paper. All authors read and approved the final manuscript.

\section{Acknowledgements}

The second author was supported by the Thailand Research Fund and the King Mongkut's University of Technology Thonburi (Grant No. RSA5780059)

Received: 4 July 2014 Accepted: 6 November 2014 Published: 26 Nov 2014

\section{References}

1. Youla, DC, Webb, H: Image restoration by the method of convex projections: part 1 - theory. IEEE Trans. Med. Imaging 1, 81-94 (1982)

2. Kitahara, S, Takahashi, W: Image recovery by convex combinations of sunny nonexpansive retractions. Topol. Methods Nonlinear Anal. 2(2), 333-342 (1993) 
3. Takahashi, W, Tamura, T: Limit theorems of operators by convex combinations of nonexpansive retractions in Banach spaces. J. Approx. Theory 91(3), 386-397 (1997)

4. Kimura, Y, Nakajo, K: The problem of image recovery by the metric projections in Banach spaces. Abstr. Appl. Anal. 2013, Article ID 817392 (2013)

5. Alber, Yl: Metric and generalized projections operators in Banach spaces: properties and applications. In: Kartsatos, AG (ed.) Theory and Applications of Nonlinear Operators of Accretive and Monotone Type. Lecture Notes in Pure and Applied Mathematics, vol. 178, pp. 15-50. Dekker, New York (1996)

6. Alber, YI, Reich, S: An iterative method for solving a class of nonlinear operator equations in Banach spaces. Panam. Math. J. 4(2), 39-54 (1994)

7. Kamimura, S, Takahashi, W: Strong convergence of a proximal-type algorithm in a Banach space. SIAM J. Optim. 13(3), 938-945 (2002)

8. Nakajo, K, Shimoji, K, Takahashi, W: Strong convergence theorems by the hybrid method for families of mappings in Banach spaces. Nonlinear Anal., Theory Methods Appl. 71(3-4), 812-818 (2009)

9. Kimura, Y, Nakajo, K, Takahashi, W: Strongly convergent iterative schemes for a sequence of nonlinear mappings. J. Nonlinear Convex Anal. 9(3), 407-416 (2008)

10. Kohsaka, F, Takahashi, W: Iterative scheme for finding a common point of infinitely many convex sets in a Banach space. J. Nonlinear Convex Anal. 5(3), 407-414 (2004)

11. Matsushita, S, Takahashi, W: A strong convergence theorem for relatively nonexpansive mappings in a Banach space. J. Approx. Theory 134(2), 257-266 (2005)

12. Matsushita, S, Nakajo, K, Takahashi, W: Strong convergence theorems obtained by a generalized projections hybrid method for families of mappings in Banach spaces. Nonlinear Anal., Theory Methods Appl. 73(6), 1466-1480 (2010)

13. Sunthrayuth, P, Kumam, P: Iterative methods for variational inequality problems and fixed point problems of a countable family of strict pseudo-contractions in a q-uniformly smooth Banach space. Fixed Point Theory Appl. 2012, 65 (2012)

14. Song, Y, Ceng, L-C: A general iteration scheme for variational inequality problem and common fixed point problems of nonexpansive mappings in q-uniformly smooth Banach spaces. J. Glob. Optim. (2013). doi:10.1007/s10898-012-9990-4

15. Cholamjiak, P, Suantai, S: Strong convergence for a countable family of strict pseudocontractions in $q$-uniformly smooth Banach spaces. Comput. Math. Appl. 62, 787-796 (2011)

16. Zhang, H, Su, Y: Strong convergence theorems for strict pseudo-contractions in q-uniformly smooth Banach spaces. Nonlinear Anal. 70, 3236-3242 (2009)

17. Cai, G, Hu, CS: Strong convergence theorems of a general iterative process for a finite family of $\boldsymbol{\lambda}_{j}$-strict pseudo-contractions in q-uniformly smooth Banach spaces. Comput. Math. Appl. 59, 149-160 (2010)

18. Song, Y, Hu, H, Ceng, LC: On variational inclusion and common fixed point problems in q-uniformly smooth Banach spaces. J. Appl. Math. 2012, Article ID 865810 (2012)

19. Cai, G, Bu, S: An iterative algorithm for a general system of variational inequalities and fixed point problems in q-uniformly smooth Banach spaces. Optim. Lett. 7(2), 267-287 (2013)

20. Zhou, HY: Convergence theorems for $\lambda$-strict pseudo-contractions in q-uniformly smooth Banach spaces. Acta Math. Sin. Engl. Ser. 26(4), 743-758 (2010)

21. Zhang, $\mathrm{H}, \mathrm{Su}, \mathrm{Y}$ : Convergence theorems for strict pseudo-contractions in q-uniformly smooth Banach spaces. Nonlinear Anal. 71, 4572-4580 (2009)

22. Saewan, S, Kumam, P: A strong convergence theorem concerning a hybrid projection method for finding common fixed points of a countable family of relatively quasi-nonexpansive mappings. J. Nonlinear Convex Anal. 13(2), 313-330 (2012)

23. Saewan, S, Kumam, P: Computational of generalized projection method for maximal monotone operators and a countable family of relatively quasi-nonexpansive mappings. Optimization (2013). doi:10.1080/02331934.2013.824444

24. Saewan, S, Kumam, P, Kanjanasamranwong, P: The hybrid projection algorithm for finding the common fixed points of nonexpansive mappings and the zeroes of maximal monotone operators in Banach spaces. Optimization (2013). doi:10.1080/02331934.2012.724686

25. Marino, G, Xu, HK: A general iterative method for nonexpansive mappings in Hilbert spaces. J. Math. Anal. Appl. 318 43-52 (2006)

26. Yamada, I: The hybrid steepest descent method for the variational inequality problems over the intersection of fixed point sets of nonexpansive mappings. In: Butnariu, D, Censor, Y, Reich, S (eds.) Inherently Parallel Algorithms in Feasibility and Optimization and Their Applications. Stud. Comput. Math., vol. 8, pp. 473-504. North-Holland, Amsterdam (2001)

27. Tian, M: A general iterative algorithm for nonexpansive mappings in Hilbert spaces. Nonlinear Anal., Theory Methods Appl. 73, 689-694 (2010)

28. Ceng, L-C, Guu, S-M, Yao, J-C: A general composite iterative algorithm for nonexpansive mappings in Hilbert spaces. Comput. Math. Appl. 61, 2447-2455 (2011)

29. Takahashi, W: Nonlinear Functional Analysis. Yokohama Publishers, Yokohama (2000)

30. Xu, ZB, Roach, GF: Characteristic inequalities of uniformly smooth Banach spaces. J. Math. Anal. Appl. 157, 189-210 (1991)

31. Takahashi, Y, Hashimoto, K, Kato, M: On sharp uniform convexity, smoothness, and strong type, cotype inequalities. J. Nonlinear Convex Anal. 3, 267-281 (2002)

32. Browder, FE, Petryshyn, WV: Construction of fixed points of nonlinear mappings in Hilbert space. J. Math. Anal. Appl. 20, 197-228 (1967)

33. Chen, $\mathrm{R}$, Song, $\mathrm{Y}$, Zhou, $\mathrm{H}$ : Convergence theorems for implicit iteration process for a finite family of continuous pseudocontractive mappings. J. Math. Anal. Appl. 314, 701-709 (2006)

34. Reich, S: Asymptotic behavior of contractions in Banach spaces. J. Math. Anal. Appl. 44, 57-70 (1973)

35. Mitrinović, DS: Analytic Inequalities. Springer, New York (1970)

36. Xu, HK: Inequalities in Banach spaces with applications. Nonlinear Anal., Theory Methods Appl. 16, 1127-1138 (1991) 
37. Dong, Q-L, He, S, Su, F: Strong convergence of an iterative algorithm for an infinite family of strict pseudo-contractions in Banach spaces. Appl. Math. Comput. 216, 959-969 (2010)

38. Opial, Z: Weak convergence of the sequence of successive approximations for nonexpansive mappings. Bull. Am. Math. Soc. 73, 591-597 (1967)

39. Agarwal, RP, O'Regan, D, Sahu, DR: Fixed Point Theory for Lipschitzian-Type Mappings with Applications. Springer, Berlin (2009)

40. Liu, LS: Iterative processes with errors for nonlinear strongly accretive mappings in Banach spaces. J. Math. Anal. Appl. $75,114-292(1980)$

10.1186/1029-242X-2014-473

Cite this article as: Sunthrayuth and Kumam: Fixed point solutions for variational inequalities in image restoration over q-uniformly smooth Banach spaces. Journal of Inequalities and Applications 2014, 2014:473

Submit your manuscript to a SpringerOpen ${ }^{\circ}$ journal and benefit from:

- Convenient online submission

- Rigorous peer review

- Immediate publication on acceptance

Open access: articles freely available online

- High visibility within the field

- Retaining the copyright to your article

Submit your next manuscript at $\gg$ springeropen.com 